\title{
Graded Manifolds and Drinfeld Doubles for Lie
} Bialgebroids

Voronov, Theodore

2002

MIMS EPrint: 2006.348

Manchester Institute for Mathematical Sciences

School of Mathematics

The University of Manchester

\footnotetext{
Reports available from: http://eprints.maths.manchester.ac.uk/

And by contacting: The MIMS Secretary

School of Mathematics

The University of Manchester

Manchester, M13 9PL, UK
} 


\title{
Graded manifolds and Drinfeld doubles for Lie bialgebroids
}

\author{
Theodore Voronov
}

\begin{abstract}
We define graded manifolds as a version of supermanifolds endowed with an extra $\mathbb{Z}$-grading in the structure sheaf, called weight (not linked with parity). Examples are ordinary supermanifolds, vector bundles, double vector bundles (in particular, iterated constructions like TTM), etc. I give a construction of doubles for graded $Q S$ - and graded QP-manifolds (graded manifolds endowed with a homological vector field and a Schouten/Poisson bracket). Relation is explained with Drinfeld's Lie bialgebras and their doubles. Graded $Q S$-manifolds can be considered, roughly, as "generalized Lie bialgebroids". The double for them is closely related with the analog of Drinfeld's double for Lie bialgebroids recently suggested by Roytenberg. Lie bialgebroids as a generalization of Lie bialgebras, over some base manifold, were defined by Mackenzie and P. Xu. Graded $Q P$-manifolds give an odd version for all this, in particular, they contain "odd analogs" for Lie bialgebras, Manin triples, and Drinfeld's double.
\end{abstract}

\section{Introduction}

Analog for Lie bialgebroids of Drinfeld's classical double is a puzzle. Various constructions were suggested (Mackenzie, Liu-Weinstein-Xu and Roytenberg), all different and all giving something which is not a Lie bialgebroid, even not a Lie algebroid. Recall that the Drinfeld double of a Lie bialgebra is again a Lie bialgebra (with nice properties). In Roytenberg's thesis 22 it was suggested to consider the supermanifold $T^{*}(\Pi E)$ (with a particular natural structure) as the "right" notion of the double for a Lie bialgebroid $E \rightarrow X$ over a base $X$. (Close ideas are due to A. Vaintrob 26.)

In this paper we define a class of supermanifolds which, in particular, generalizes the structure of Lie bialgebroids. We prove that an analog of Roytenberg's construction carries over for them. Namely, we consider a "graded manifold" (see below) $M$ endowed with a homological vector field $\hat{Q}$ of weight $q$ and a compatible Schouten bracket of weight $s$, and prove that on the space of the cotangent bundle $T^{*} M$ there is a naturally defined homological field $\hat{Q}_{D}$ and it is possible to introduce weight for $T^{*} M$ in such a way that $\hat{Q}_{D}$ will be again of the weight $q$ (same

2000 Mathematics Subject Classification. Primary: 53D17, 58A50; secondary: 17B62, $17 \mathrm{~B} 63$.

Key words and phrases. Graded manifolds, Lie bialgebroids, supermanifolds, Poisson brackets, Schouten brackets, homological vector fields, Drinfeld double, odd double, odd Lie bialgebras.

The research was supported in part by EPSRC, under grant GR/N00821/01. 
as original $\hat{Q}$ ), and certain natural properties hold. The space $D M=T^{*} M$ with such a structure is called the double of $M$. The double $D M$ so defined inherits half the original structure of $M$, a homological field. Using a linear connection on $M$, it is possible to define on $D M$ an "almost" Schouten bracket as well. I show that this exactly recovers the whole structure of the Drinfeld double when restricted to Lie bialgebras. (For a nonlinear $M$, the bracket on $D M$ does not, in general, obey the Jacobi identity.) By considering supermanifolds with a Poisson rather than Schouten bracket, we obtain an odd analog of this theory. We introduce and study odd Lie bialgebras and odd double for them. In particular, we construct an odd Lie bialgebra structure in the Lie superalgebra $\mathfrak{q}(n)$. (A quantization of this example should give a quantum supergroup $G Q_{\varepsilon}(n)$ with an odd quantum parameter $\varepsilon$.)

This work is based on two simple ideas.

The first is a key notion of a graded manifold. Probably, the concept is "in the air". Needless to argue for the usefulness of $\mathbb{Z}$-grading in algebraic questions. Unfortunately, there is an often confusion with $\mathbb{Z}_{2}$-grading responsible for the sign rule. Of course, in many examples the $\mathbb{Z}_{2}$-grading is induced by some underlying $\mathbb{Z}$-grading, but in other examples not, and a natural $\mathbb{Z}$-grading can have nothing to do with the sign rule (example: degree for polynomials). We introduce graded manifolds as a certain version of supermanifolds with a $\mathbb{Z}$-grading in the structure sheaf independent of parity. We call it weight. This structure embraces many examples and carries over to natural constructions. (The term "graded manifolds" was used in some early works on supermanifolds instead of the latter term. Our usage has no relation with this.)

The second is a "geometrization" of the concept of a Lie algebra, in the following sense. (Everywhere when we say "Lie algebra" we mean "Lie superalgebra".) This is the answer to a simple question: to which structure in the algebra of functions corresponds the Lie bracket? Consider simultaneously the vector space $\mathfrak{g}$ with all its "neighbors": $\mathfrak{g}^{*}, \Pi \mathfrak{g}, \Pi \mathfrak{g}^{*}$. On $\mathfrak{g}^{*}$ we get a linear Poisson bracket (the Lie-Berezin-Kirillov bracket). On $\Pi \mathfrak{g}^{*}$ we get a linear odd Poisson (=Schouten, Gerstenhaber) bracket, which we call the Lie-Schouten bracket. On $\Pi \mathfrak{g}$ we get a quadratic homological vector field $Q= \pm(1 / 2) c_{i j}^{k} \xi^{j} \xi^{i} \partial / \partial \xi^{k}$. Hence, Poisson brackets, Schouten brackets and homological vector fields are all, equally, extensions of a Lie algebra structure. We call supermanifolds with such structures $P$-manifolds, $S$-manifolds and $Q$-manifolds, respectively ${ }^{1}$. The next question is, what is a "bi-" structure from this viewpoint. Shortly, it is a Lie algebra structure on two neighbors with a compatibility condition. We get the following list: $\left(\mathfrak{g}, \mathfrak{g}^{*}\right)$, which is a Lie bialgebra in Drinfeld's sense, $\left(\mathfrak{g}, \Pi \mathfrak{g}^{*}\right)$, which is the odd analog of Drinfeld's notion (can be non-trivial only for superalgebras), and $(\mathfrak{g}, \Pi \mathfrak{g})$. Their geometric versions are, respectively: $Q S$-manifolds (compatible $Q$ - and $S$-structures), $Q P$-manifolds (compatible $Q$ - and $P$-structures), and PS-manifolds (two compatible brackets of the opposite parity; structures of this kind have very interesting geometry, their study was initiated in $\mathbf{7}$ ). Notice, that for a "bi-" Lie structure of each type there is just one geometric manifestation (one type of structure, though it can be realized on different spaces, e.g., the $Q S$-structures on $\Pi \mathfrak{g}$ and $\Pi \mathfrak{g}^{*}$ for a Drinfeld

\footnotetext{
${ }^{1}$ Curiously, the most difficult is to tell what structure in the algebra of functions on $\mathfrak{g}$ itself corresponds to the Lie bracket on $\mathfrak{g}$. An analogous question is, what natural operator exists on functions on $T M$, i.e., on Lagrangians. Partial answer, of course, is: the Euler-Lagrange equations. Their analog for Lie algebras and Lie algebroids was considered by A. Weinstein 35 .
} 
Lie bialgebra $\mathfrak{g}$ ), compared to the three different manifestations of a "single" Lie bracket.

Combining these two ideas, we say that instead of conventional Lie (bi)algebroids one should deal with graded manifolds with $Q S$ - and $Q P$-structure and keep track of weights. In particular, this allows to develop a nice framework for doubles. As a byproduct, we naturally encounter odd analogs for Lie bialgebras and bialgebroids.

The paper is organized as follows:

In Section 1 we review the necessary facts concerning Poisson and Schouten algebras. In particular, it is shown how an arbitrary Poisson structure is expressed via the canonical Schouten structure on the anticotangent bundle, and an arbitrary Schouten structure is expressed via the canonical Poisson bracket on the cotangent bundle. We see how even and odd brackets intertwine.

In Section 2 we give a geometric description of Drinfeld's Lie bialgebras, in the language of supermanifolds, and we explain how the classical Drinfeld's double for $\mathfrak{g}$ is equivalent to a natural construction of a $Q S$-structure on $T^{*} \Pi \mathfrak{g} \cong \Pi\left(\mathfrak{g} \oplus \mathfrak{g}^{*}\right)$. This also leads us to a new notion of an odd Lie bialgebra, which we define in terms of a $Q P$-structure on $\Pi \mathfrak{g}$.

In Section 3 we review the "super" approach to Lie bialgebroids and doubles. It is a generalization of the "super" description of Lie bialgebras and their Drinfeld doubles given in Section 2. We describe Roytenberg's "Drinfeld double" of a Lie bialgebroid. It is no longer a Lie algebroid. Actually, it is an example of a graded manifold (which we define in the next section).

In Section 4 we introduce graded manifolds as supermanifolds with a "weight" in the algebra of functions, and show how it carries over to natural bundles and the canonical brackets. In particular, we show that the natural bundles like $T M$ are bi-graded. This gives important flexibility for the definition of a "total" weight.

In Section 5 we consider graded $Q S$ - and $Q P$-manifolds. The former should be regarded as generalized Lie (anti)bialgebroids. The latter are a generalization of odd Lie bialgebras defined in Section 2. For both we give a construction of a double as a graded $Q$-manifold. For a graded $Q S$-manifold $\Pi E$, where $E$ is a Lie bialgebroid, this recovers Roytenberg's construction. We show how with the help of a connection it is possible to construct an "almost" bracket in the double.

In Section 6 we consider odd Lie bialgebras and the odd double in detail. We consider as example the matrix superalgebra $\mathfrak{q}(n)$ and show that it has a natural odd bialgebra structure. As a technical tool to define this structure, we introduce a "relative" version of doubles, in the situation when there is an action of some Lie superalgebra. (This models certain triangular decompositions of superalgebras.)

In Appendix we give a proof of a theorem of Mackenzie and $\mathrm{Xu}$ about cotangent bundles for dual vector bundles. We also suggest and prove its odd analog.

Note about usage. All our constructions are "superized", i.e., we automatically work with supermanifolds, superalgebras, super Lie (bi)algebroids, etc., from the beginning. "Algebras" and "manifolds" stand for "superalgebras" and "supermanifolds" throughout the text unless otherwise required for clarity. $\Pi$ stands for the parity reversion functor. We use the prefix "anti" for opposite vector spaces and vector bundles (spaces and bundles with reversed parity). For example, anticotangent bundle means $\Pi T^{*} M$, Lie antialgebra means the space $\Pi \mathfrak{g}$ for a Lie algebra $\mathfrak{g}$, an antivector (for a vector space $V$ ) means an element of $\Pi V$. 


\section{Poisson and Schouten algebras}

For the sake of completeness, we provide some definitions and facts. All vector spaces are assumed $\mathbb{Z}_{2}$-graded, with tilde denoting parity of a homogeneous element. Commutators, commutativity, derivations, linearity, etc., are understood in the $\mathbb{Z}_{2^{-}}$ graded sense. The numbers 0,1 denoting the grading are residues modulo 2 .

Definition 1.1. A Poisson algebra is a vector space $A=A_{0} \oplus A_{1}$ with a bilinear associative multiplication $(a, b) \mapsto a b$ such that $A_{i} A_{j} \subset A_{i+j}$ and an even bilinear operation denoted $\{$,$\} (i.e., \left\{A_{i}, A_{j}\right\} \subset A_{i+j}$ ) satisfying

$$
\begin{aligned}
\{a, b\} & =-(-1)^{\tilde{a} \tilde{b}}\{b, a\} \\
\{a,\{b, c\}\} & =\{\{a, b\}, c\}+(-1)^{\tilde{a} \tilde{b}}\{b,\{a, c\}\} \\
\{a, b c\} & =\{a, b\} c+(-1)^{\tilde{a} \tilde{b}} b\{a, c\}
\end{aligned}
$$

for every homogeneous elements $a, b, c \in A$. The operation $\{$,$\} is called Poisson$ bracket.

Definition 1.2. A Schouten algebra is a vector space $A=A_{0} \oplus A_{1}$ with a bilinear associative multiplication $(a, b) \mapsto a b$ such that $A_{i} A_{j} \subset A_{i+j}$ and an odd bilinear operation denoted $\{$,$\left.\} (i.e., \left\{A_{i}, A_{j}\right\} \subset A_{i+j+1}\right)$ satisfying

$$
\begin{aligned}
\{a, b\} & =-(-1)^{(\tilde{a}+1)(\tilde{b}+1)}\{b, a\} \\
\{a,\{b, c\}\} & =\{\{a, b\}, c\}+(-1)^{(\tilde{a}+1)(\tilde{b}+1)}\{b,\{a, c\}\} \\
\{a, b c\} & =\{a, b\} c+(-1)^{(\tilde{a}+1) \tilde{b}} b\{a, c\}
\end{aligned}
$$

for every homogeneous elements $a, b, c \in A$. The operation $\{$,$\} is called Schouten$ bracket.

The difference between these two notions is obvious. A Poisson algebra is, in particular, a Lie (super)algebra, and the identity (1.3) tells that for every $a \in A$, the operator ad $a=\{a$,$\} acts as a derivation of the associative product. The operator$ ad $a$ has the same parity as $a$. Similarly, a Schouten algebra is, in particular, a Lie superalgebra, but w.r.t. the reversed parity. The operator ad $a:=(-1)^{\tilde{a}+1}\{a$,$\} in$ a Schouten algebra is a derivation of the associative product of parity opposite to that of $a$. No shift of parity can turn (1.6) into (1.3).

Other names for Schouten algebras are odd Poisson algebras and Gerstenhaber algebras. Schouten bracket is also known as odd bracket and antibracket.

REMARK 1.1. Schouten algebras became very popular recently, in connection with their use in Batalin-Vilkovisky (BV) formalism in quantum field theory [1, 2 and in deformation quantization (see, e.g., [8] ). The name "Gerstenhaber algebras" is motivated by the corresponding structure discovered by Gerstenhaber [4] in the cohomology of an associative algebra. The name "Schouten algebra" comes from the canonical Schouten bracket of multivector fields [23, see below. This bracket is completely parallel with the canonical Poisson bracket on $T^{*} M$, so the choice of "Schouten algebras" for the odd counterpart of "Poisson algebras" seems natural. For a manifold provided with a volume element there is another operation on multivector fields, the divergence. It satisfies the relation

$$
\delta(P Q)=\delta P Q+(-1)^{\tilde{P}} P \delta Q+(-1)^{\tilde{P}+1}\{P, Q\},
$$


thus connecting Schouten bracket and multiplication. Schouten algebras endowed with a differential satisfying (1.7) received the name Batalin-Vilkovisky algebras (BV-algebras), because of their role in the BV-formalism.

Definition 1.3. A (super)manifold with a Poisson (Schouten) structure in the algebra of functions is called a Poisson (resp., Schouten or odd Poisson) manifold.

Basic examples: $T^{*} M$ and the canonical Poisson bracket on functions on $T^{*} M$ (Hamiltonians on $M$ ), $\Pi T^{*} M$ and the canonical Schouten bracket on functions on $\Pi T^{*} M$ (multivector fields on $M$ ). They solve two universal problems: for Lie homomorphisms Vect $M \rightarrow A$ to commutative Poisson algebras "over $C^{\infty}(M)$ " and for odd Lie homomorphisms Vect $M \rightarrow A$ to commutative Schouten algebras "over $C^{\infty}(M)$ " (precise formulation requires the notions of Lie-Rinehart algebras or Lie algebroids, see below). In the sequel we will use the following notation for the homomorphisms from vector fields to Hamiltonians and to multivector fields: $p: X \mapsto p_{X}$ and $\theta: X \mapsto \theta_{X}$, respectively. (Notice that the second map is odd.)

A purely algebraic variant is given by the Poisson bracket on $C^{\infty}\left(\mathfrak{g}^{*}\right)$,

$$
\left\{x_{i}, x_{j}\right\}=c_{i j}^{k} x_{k},
$$

for a Lie algebra $\mathfrak{g}$, the Lie-Poisson(-Berezin-Kirillov) bracket, and by the Schouten bracket on $C^{\infty}\left(\Pi \mathfrak{g}^{*}\right)$,

$$
\left\{\xi_{i}, \xi_{j}\right\}=c_{i j}^{k} \xi_{k}
$$

(where the coordinates $\xi_{i}$ have parity opposite to that of the respective $x_{i}$ ), the LieSchouten bracket, as we shall call it. They solve universal problems for even/odd bracket-preserving maps $\mathfrak{g} \rightarrow A$ to commutative Poisson/Schouten algebras respectively. (For the even case this is directly related with the moment map for Hamiltonian actions of $\mathfrak{g}$.)

Examples above are united in the concept of a Lie algebroid (see [21, 18]). A Lie algebroid is a vector bundle $E \rightarrow M$ over a manifold $M$ endowed with a vector bundle map $a: E \rightarrow T M$ (called the anchor) and a Lie algebra structure on the space of sections $C^{\infty}(M, E)$ satisfying

$$
\begin{aligned}
{[u, f v] } & =a(u) f v+(-1)^{\tilde{u} \tilde{f}} f[u, v] \\
a([u, v]) & =[a(u), a(v)]
\end{aligned}
$$

for all $u, v \in C^{\infty}(M, E)$ and all $f \in C^{\infty}(M)$. Here we identify vector fields on $M$ with derivations of the algebra $C^{\infty}(M)$. Likewise, for an arbitrary (commutative associative) algebra $A$, a Lie pseudoalgebra or a Lie-Rinehart algebra over $A$ is a module $L$ over $A$ with a Lie algebra structure and a homomorphism of modules $a: L \rightarrow \operatorname{Der} A$ satisfying (1.10),(1.11) (see [18]).

It is not difficult to see that every Lie algebroid (or every Lie-Rinehart algebra) canonically produces two algebras, a Poisson algebra and a Schouten algebra. The Poisson algebra associated with a Lie algebroid $E$ is $C^{\infty}\left(E^{*}\right)$. The Schouten algebra associated with $E$ is $C^{\infty}\left(\Pi E^{*}\right)$. Axioms of Poisson/Schouten algebra for $C^{\infty}\left(E^{*}\right)$, $C^{\infty}\left(\Pi E^{*}\right)$ are equivalent to $E$ being a Lie algebroid. These algebras are universal in a certain precise sense, and their construction unites the constructions of the canonical Poisson bracket in $C^{\infty}\left(T^{*} M\right)$ and the canonical Schouten bracket in $C^{\infty}\left(\Pi T^{*} M\right)$ from the commutator of vector fields with the constructions of the Lie-Poisson bracket in $C^{\infty}\left(\mathfrak{g}^{*}\right)$ and the Lie-Schouten bracket in $C^{\infty}\left(\Pi \mathfrak{g}^{*}\right)$ from the bracket in $\mathfrak{g}$. 
Most often the associative multiplication in the definition of Poisson/Schouten algebras is assumed to be commutative. There are natural examples where it is not so. The commutator bracket $[a, b]=a b-(-1)^{\tilde{a} \tilde{b}} b a$ obviously satisfies the Leibniz identity (1.3) and thus makes every associative algebra into a (noncommutative) Poisson algebra. Let us reserve the notation [, ] for the commutator bracket and denote a given arbitrary Poisson bracket by $\{$,$\} .$

Lemma 1.1 (32]). In every Poisson algebra the following identity holds:

$$
[a, b]\{c, d\}=\{a, b\}[c, d]
$$

for arbitrary elements $a, b, c, d$.

Obviously, (1.12) degenerates in two special cases, when the algebra is commutative and when the bracket coincides with the commutator, as in the example above.

Consider a Lie algebra $\mathfrak{g}$. Let us denote the Lie brackets in $\mathfrak{g}$ by $\{$,$\} . One may ask about$ bracket-preserving maps $\mathfrak{g} \rightarrow A$ to Poisson algebras without conditions of commutativity.

TheOrem 1.1 ([32]). A non-commutative Poisson algebra that is a universal range for such maps (the "Poisson envelope" of a Lie algebra $\mathfrak{g}$ ) is given by $E(\mathfrak{g}):=T(\mathfrak{g}) / I$ where the ideal I in the free tensor algebra is generated by the elements of the form

$$
a \otimes b-(-1)^{\tilde{a} \tilde{b}} b \otimes a \otimes\{c, d\}-\{a, b\} \otimes c \otimes d-(-1)^{\tilde{c} \tilde{d}} d \otimes c
$$

for all $a, b, c, d \in \mathfrak{g}$.

The algebra $C_{\text {pol }}^{\infty}\left(\mathfrak{g}^{*}\right)$ and the universal enveloping algebra $U(\mathfrak{g})$ both are quotients of $E(\mathfrak{g})$. The algebra $E(\mathfrak{g})$ can be viewed as a non-commutative analog of the Kirillov-Kostant-Sourieau moment space $\mathfrak{g}^{*}$. For details see $\mathbf{3 2}$.

On a manifold $M$, a non-degenerate bracket is specified by a symplectic 2form $\omega \in \Omega^{2}(M)$, even for the Poisson case and odd for the Schouten case. The correspondence is given by the formulas: $i_{X_{f}} \omega=-(-1)^{s \tilde{f}} d f,\{f, g\}=(-1)^{s \tilde{f}} X_{f} g$, $X_{f}$ being the Hamiltonian vector field corresponding to a function $f$, where $s=0$ for an even bracket and $s=1$ for an odd bracket.

Consider the general situation. From $(1.3),(1.1),(1.6),(1.4)$ follows that a Poisson as well as a Schouten bracket is a bi-derivation of functions. Hence a bracket is specified by a contravariant tensor field of rank 2 . From antisymmetry follows that in the Poisson case it should be an even bivector field. What about the Schouten case? Contrary to what one might think at the first moment, an odd bracket is not related with odd bivector fields. In fact, a Schouten bracket on $M$ is specified by a fiberwise quadratic odd Hamiltonian function ${ }^{2}$. Given an even bivector field $P \in C^{\infty}\left(\Pi T^{*} M\right)$, a Poisson bracket $\{f, g\}_{P}$ of functions $f$ and $g$ is defined by the coordinate-free formula

$$
\{f, g\}_{P}:=\{f,\{P, g\}\}
$$

where the brackets at the r.h.s. are the canonical Schouten brackets of multivector fields. The Jacobi identity for $\{,\}_{P}$ is equivalent to the identity $\{P, P\}=0$. Likewise, given an odd quadratic Hamiltonian $S \in C^{\infty}\left(T^{*} M\right)$, it defines a Schouten structure $\{,\}_{S}$ by the similar formula

$$
\{f, g\}_{S}:=\{f,\{S, g\}\}
$$

where at the r.h.s. there are the canonical Poisson brackets, and the Jacobi identity for $\{,\}_{S}$ is equivalent to the identity $\{S, S\}=0$. The interrelation between Poisson

\footnotetext{
${ }^{2}$ In a non-degenerate situation, brackets of both types are specified by 2-forms. Noteworthy, the inverse matrix for the components of such a form possesses different symmetry properties depending on its parity, corresponding, up to certain sign factors, to the components of a bivector for an even form and to the coefficients of a quadratic function for an odd form.
} 
and Schouten structures is summarized in the table below (see also Appendix B to [29]). Notice that $\{f,\{P, g\}\}=\{\{f, P\}, g\}$, and $\{f,\{S, g\}\}=\{\{f, S\}, g\}$.

\begin{tabular}{|c|c|}
\hline A Poisson manifold $M$ & A Schouten manifold $M$ \\
\hline $\multicolumn{2}{|c|}{f, g}_{P, S}=(-1)^{\tilde{a}(\tilde{f}+1)} \frac{\partial f}{\partial x^{a}}\left\{x^{a}, x^{b}\right\}_{P, S} \frac{\partial g}{\partial x^{b}}$ \\
(same coordinate formula for even and odd bracket)
\end{tabular}

(Notation: $x^{a}$ stand for local coordinates on $M, p_{a}$ for the induced coordinates in the cotangent space, $x_{a}^{*}$ for the induced coordinates in the anticotangent space. Here $\tilde{x}^{a}=\tilde{a}=\tilde{p}_{a}, \tilde{x}_{a}^{*}=\tilde{a}+1$. The transformation law for $p_{a}$ and $x_{a}^{*}$ is the same.)

We shall later need the coordinate formulas for the canonical brackets.

On $T^{*} M$ the canonical even symplectic form is $d p_{a} d x^{a}=d\left(d x^{a} p_{a}\right)=d\left(p_{a} d x^{a}\right)$. The corresponding canonical Poisson bracket:

$$
\{f, g\}=(-1)^{\tilde{a}(\tilde{f}+1)} \frac{\partial f}{\partial p_{a}} \frac{\partial g}{\partial x^{a}}-(-1)^{\tilde{a} \tilde{f}} \frac{\partial f}{\partial x^{a}} \frac{\partial g}{\partial p_{a}},
$$

where $f, g \in C^{\infty}\left(T^{*} M\right)$, i.e., Hamiltonians on $M$. In particular, $\left\{p_{a}, x^{b}\right\}=\delta_{a}^{b}=$ $-(-1)^{\tilde{a}}\left\{x^{b}, p_{a}\right\}$.

On $\Pi T^{*} M$ the canonical odd symplectic form is $(-1)^{\tilde{a}+1} d x_{a}^{*} d x^{a}=d\left(d x^{a} x_{a}^{*}\right)=$ $d\left((-1)^{\tilde{a}+1} x_{a}^{*} d x^{a}\right)$. The corresponding canonical Schouten bracket:

$$
\{f, g\}=(-1)^{(\tilde{a}+1)(\tilde{f}+1)} \frac{\partial f}{\partial x_{a}^{*}} \frac{\partial g}{\partial x^{a}}-(-1)^{\tilde{a}(\tilde{f}+1)} \frac{\partial f}{\partial x^{a}} \frac{\partial g}{\partial x_{a}^{*}},
$$

where $f, g \in C^{\infty}\left(\Pi T^{*} M\right)$ (multivector fields on $\left.M\right)$. In particular, $\left\{x_{a}^{*}, x^{b}\right\}=\delta_{a}^{b}=$ $-\left\{x^{b}, x_{a}^{*}\right\}$.

The properties of the brackets $(1.14)$ and $(1.15)$ are derived from the properties of the canonical brackets. Crucial is that the functions on $M$ pulled-back to $T^{*} M$ or $\Pi T^{*} M$ have vanishing canonical brackets. In particular, this implies antisymmetry of a new bracket. In such description it is clear that this construction can be put into an abstract setting and generalized (in particular, iterated). It goes as follows.

Definition 1.4 (see [11, [12]). A Loday algebra of parity $s=0,1$, is a vector space $L$ endowed with a bilinear operation of parity $s$ (a Loday bracket) satisfying 
the condition

$$
[a,[b, c]]=[[a, b] c]+(-1)^{(\tilde{a}+s)(\tilde{b}+s)}[b,[a, c]] .
$$

In other words, a Loday algebra is a crippled Lie superalgebra without the antisymmetry condition and where the Jacobi identity remains in the form of a one-sided "Leibniz identity" for the bracket. (Loday [16] introduced these algebras under the name of "Leibniz algebras".)

Consider an odd derivation $D$ of a Loday algebra $L$. Introduce a new operation $[a, b]_{D}:=(-1)^{\tilde{a}}[D a, b]$. Obviously, it is a bilinear operation of parity $s+1$.

THEOREM $1.2([\mathbf{1 1}])$. If $D^{2}=0$, then the bracket $[,]_{D}$ defines a new structure of a Loday algebra on the space $L$, of parity $s+1$.

Proof. Consider

$$
\begin{aligned}
& {\left[a,[b, c]_{D}\right]_{D} }=(-1)^{\tilde{a}+\tilde{b}}[D a,[D b, c]]= \\
&(-1)^{\tilde{a}+\tilde{b}}\left([[D a, D b], c]+(-1)^{(\tilde{a}+s+1)(\tilde{b}+s+1)}[D b,[D a, c]]\right)= \\
&(-1)^{\tilde{a}+\tilde{b}}\left((-1)^{\tilde{a}+s+1}[D[D a, b], c]+(-1)^{(\tilde{a}+s+1)(\tilde{b}+s+1)}[D b,[D a, c]]\right),
\end{aligned}
$$

because $\left.D[D a, b]=\left[D^{2} a, b\right]+(-1)^{\tilde{a}+s+1}[D a, D b]=-1\right)^{\tilde{a}+s+1}[D a, D b]$, due to $D^{2}=$ 0 . Thus it equals (we continue)

$$
\begin{array}{r}
(-1)^{\tilde{a}+\tilde{b}+s+1}\left[D(-1)^{\tilde{a}}[D a, b], c\right]+(-1)^{(\tilde{a}+s+1)(\tilde{b}+s+1)}(-1)^{\tilde{b}}\left[D b,(-1)^{\tilde{a}}[D a, c]\right]= \\
{\left[[a, b]_{D}, c\right]_{D}+(-1)^{(\tilde{a}+s+1)(\tilde{b}+s+1)}\left[b,[a, c]_{D}\right]_{D}}
\end{array}
$$

The new bracket (of the opposite parity) $[,]_{D}$ is called a derived bracket. Suppose $L$ is a Lie algebra of parity $s$ (cf. in [32]), i.e., there is the antisymmetry condition $[a, b]=-(-1)^{(\tilde{a}+s)(\tilde{b}+s)}[b, a]$ in addition to (1.18). Without loss of generality set $s=0$. Then $[a, b]_{D}+(-1)^{(\tilde{a}+1)(\tilde{b}+1)}[b, a]_{D}=(-1)^{\tilde{a}}[D a, b]+(-1)^{\tilde{a} \tilde{b}+\tilde{a}+1}[D b, a]=$ $(-1)^{\tilde{a}}[D a, b]+[a, D b]=(-1)^{\tilde{a}} D[a, b]$. Thus on a subspace where $D[a, b]=0$ (or on a certain quotient) the derived bracket gives a genuine Lie bracket of parity $s+1$. (See 11 for details and generalizations. Without Loday algebras, derived brackets were also considered in $[\mathbf{3 0}, \mathbf{3 1}$.)

REMARK 1.2. The derived bracket construction is seen also in the formulas of the Cartan calculus, where $\left[d, i_{u}\right]=L_{u}$ and $\left[L_{u}, i_{v}\right]=(-1)^{\tilde{u}} i_{[u, v]}$ imply $i_{[u, v]}=$ $\left[i_{u},\left[d, i_{v}\right]\right]$, which is a coordinate-free relation of the structure equation for the commutator of vector fields with that for $d$.

The construction of a Poisson/Schouten bracket from the canonical brackets fits into this abstract model, with $D=\operatorname{ad} P$ or $D=\operatorname{ad} S$, respectively. If we consider an arbitrary even multivector field $P$ satisfying $\{P, P\}=0$, rather than a bivector field, then the subalgebra $C^{\infty}(M) \subset C^{\infty}\left(\Pi T^{*} M\right)$ ceases to be closed under the new bracket. Instead, we arrive at a "Loday-Poisson" structure on the whole of $C^{\infty}\left(\Pi T^{*} M\right)$. Same holds for the Schouten case, if the restriction that the Hamiltonian $S$ is quadratic in $p_{a}$ is dropped. 
REMARK 1.3. In the algebra of functions on a Schouten manifold $M$ endowed with a volume form there is a natural "odd Laplacian"

$$
\Delta f:=\frac{1}{2} \operatorname{div} X_{f},
$$

which makes it into a Batalin-Vilkovisky algebra. The formula (1.19) is due to Khudaverdian [7. It provides an invariant geometric setup for the $\Delta$-operator of the BV-formalism (see also [5],[6]). Notice an analogy with the modular field on Poisson manifolds (see [34]). For $\Pi T^{*} M$ considered with the volume form $\rho^{2}$, where $\rho$ is a volume form on $M$, the operator (1.19) coincides with the usual divergence of multivector fields $\delta$.

\section{2. "Bi-" structures and the Drinfeld double in a supermanifold framework}

Recall the following definition. For simplicity let us first consider the purely even situation. Later we will give a different approach that automatically handles the general case.

Definition 2.1 (Drinfeld). A Lie bialgebra is a Lie algebra $\mathfrak{g}$ such that the dual space $\mathfrak{g}^{*}$ is also a Lie algebra and the two structures are compatible in the sense that

$$
\delta: \mathfrak{g} \rightarrow \wedge^{2} \mathfrak{g}
$$

is a 1-cocycle of $\mathfrak{g}$ w.r.t. $\wedge^{2}$ ad, where $\delta^{*}: \wedge^{2} \mathfrak{g}^{*} \rightarrow \mathfrak{g}^{*}$ is the bracket on $\mathfrak{g}^{*}$.

If $e_{i}$ is a basis of $\mathfrak{g}, e^{i}$ the dual basis of $\mathfrak{g}^{*}$, then the definition of a bialgebra is equivalent to the following relation between the structure constants of $\mathfrak{g}$ and $\mathfrak{g}^{*}$ (the formula below is for a purely even $\mathfrak{g}$ ):

$$
c_{j k}^{i} b_{i}^{n m}-c_{j i}^{n} b_{k}^{i m}+c_{j i}^{m} b_{k}^{i n}+c_{k i}^{n} b_{j}^{i m}-c_{k i}^{m} b_{j}^{i n}=0 .
$$

Theorem 2.1 (Drinfeld). Let $\mathfrak{g}$ be a Lie bialgebra. Then on $\mathfrak{g} \oplus \mathfrak{g}^{*}$ there is a unique structure of a Lie bialgebra described by the properties:

(1) $\mathfrak{g}$ and $\mathfrak{g}^{*}$ are Lie subalgebras,

(2) The natural inner product in $\mathfrak{g} \oplus \mathfrak{g}^{*}$ is ad-invariant,

(3) The cobracket $\delta$ for $\mathfrak{g} \oplus \mathfrak{g}^{*}$ is given by the coboundary of the element $r=e_{i} \wedge e^{i} \in \wedge^{2}\left(\mathfrak{g} \oplus \mathfrak{g}^{*}\right)$.

A partial converse is given by the following theorem.

THEOREM 2.2 (Manin). Let $\mathfrak{a}, \mathfrak{b}$ be (finite-dimensional) Lie algebras such that the vector space $\mathfrak{d}:=\mathfrak{a} \oplus \mathfrak{b}$ has a structure of a Lie algebra with an invariant inner product. Suppose that

(1) $\mathfrak{a}$ and $\mathfrak{b}$ are isotropic subspaces in $\mathfrak{d}$,

(2) $\mathfrak{a}$ and $\mathfrak{b}$ as Lie algebras are subalgebras in $\mathfrak{d}$.

Then $\mathfrak{b} \cong \mathfrak{a}^{*}$, and $\mathfrak{a}, \mathfrak{b} \cong \mathfrak{a}^{*}$ are dual Lie bialgebras. The Lie algebra structure on $\mathfrak{d}$ coincides with the one given by Drinfeld's theorem.

The space $\mathfrak{d}=\mathfrak{d}(\mathfrak{g}):=\mathfrak{g} \oplus \mathfrak{g}^{*}$ with the bialgebra structure is called the (classical) Drinfeld double of the bialgebra $\mathfrak{g}$; the triple of Lie algebras $(\mathfrak{a}, \mathfrak{b}, \mathfrak{d}=\mathfrak{a} \oplus \mathfrak{b})$ is called 
a Manin triple. Explicit formulas for the bracket in $\mathfrak{d}$ :

$$
\begin{aligned}
& {\left[e_{i}, e_{j}\right]=c_{i j}^{k} e_{k},} \\
& {\left[e^{i}, e^{j}\right]=b_{k}^{i j} e^{k},} \\
& {\left[e_{i}, e^{j}\right]=b_{i}^{j k} e_{k}-c_{i k}^{j} e^{k} .}
\end{aligned}
$$

The cobracket $\delta: \mathfrak{d} \rightarrow \wedge^{2} \mathfrak{d}$ is given by

$$
\begin{aligned}
& \delta\left(e_{i}\right)=b_{i}^{j k} e_{j} \wedge e_{k} \\
& \delta\left(e^{i}\right)=-c_{j k}^{i} e^{j} \wedge e^{k} .
\end{aligned}
$$

(Due to the natural inner product, the cobracket (2.6 2.7) gives rise to the second bracket in $\mathfrak{d}:\left[e_{i}, e_{j}\right]_{(2)}=-c_{i j}^{k} e_{k},\left[e^{i}, e^{j}\right]_{(2)}=b_{k}^{i j} e^{k},\left[e_{i}, e^{j}\right]_{(2)}=0$.)

Now, to get a different formulation suitable for further generalizations, we first have to describe a Lie algebra structure geometrically. To this end, let $\mathfrak{g}=\mathfrak{g}_{0} \oplus \mathfrak{g}_{1}$ be a vector space (from now on we return to the general super case). Consider it together with its neighbors. (The term "neighbor" for super vector spaces is due to Manin.) We have a tetrahedron ${ }^{3}$.

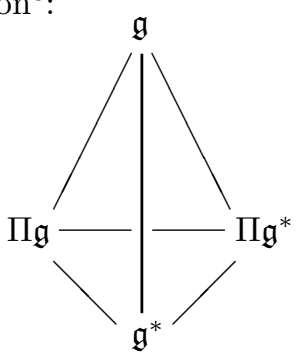

THEOREM 2.3. A Lie (super)algebra structure in the vector space $\mathfrak{g}$ is equivalent to either of:

(1) A linear Poisson bracket on the manifold $\mathfrak{g}^{*}$ (Lie coalgebra).

(2) A linear Schouten bracket on the manifold $\Pi \mathfrak{g}^{*}$ (Lie anticoalgebra).

(3) A quadratic homological vector field on the manifold $\Pi \mathfrak{g}$ (Lie antialgebra).

Recall that a homological vector field on a supermanifold $M$ is an odd vector field (=odd derivation of the algebra of functions) $\hat{Q}$ such that $[\hat{Q}, \hat{Q}]=2 \hat{Q}^{2}=0$. A basis $e_{i}$ in $\mathfrak{g}$ gives rise to linear coordinates on the supermanifolds $\Pi \mathfrak{g}, \mathfrak{g}^{*}$, and $\Pi \mathfrak{g}^{*}$, which we denote $\xi^{i}, x_{i}$ and $\xi_{i}$, respectively. If $\tilde{e}_{i}=: \tilde{\imath}$, then $\tilde{\xi}^{i}=\tilde{\imath}+1, \tilde{x}_{i}=\tilde{\imath}$, $\tilde{\xi}_{i}=\tilde{\imath}+1$.

EXAMPLE 2.1. The homological field corresponding to a Lie superalgebra $\mathfrak{g}$ with the structure constants $c_{i j}^{k}$ (i.e., $\left.\left[e_{i}, e_{j}\right]=c_{i j}^{k} e_{k}\right)$ is

$$
\hat{Q}=\frac{1}{2}(-1)^{\tilde{\jmath}} \xi^{j} \xi^{i} c_{i j}^{k} \frac{\partial}{\partial \xi^{k}}
$$

The condition $\hat{Q}^{2}=0$ is equivalent to the Jacobi identity for $c_{i j}^{k}$. An element $X \in \mathfrak{g}$ (here we treat $\mathfrak{g}$ as a vector space, not as a manifold) corresponds to a vector field on $\Pi \mathfrak{g}$ (of parity opposite to that of $X$ ) with constant coefficients:

\footnotetext{
${ }^{3}$ The picture of a tetrahedron was suggested to me by Alan Weinstein instead of the threepointed star with center at $\mathfrak{g}$ that I used initially.
} 
$X=X^{i} e_{i} \mapsto i_{X}:=(-1)^{\tilde{X}} X^{i} \partial / \partial \xi^{i}$. The odd linear map $i: \mathfrak{g} \rightarrow \operatorname{Vect}(\Pi \mathfrak{g})$ is, obviously, injective. The Lie bracket in $\mathfrak{g}$ can be reconstructed from the commutator of vector fields on $\Pi \mathfrak{g}$ by the "derived bracket" formula:

$$
i_{[X, Y]}=\left[i_{X},\left[\hat{Q}, i_{Y}\right]\right]=\left[\left[i_{X}, \hat{Q}\right], i_{Y}\right] .
$$

Here at the l.h.s. [, ] stands for the Lie bracket in $\mathfrak{g}$ with the structure constants $c_{i j}^{k}$, and at the r.h.s. [, ] stands for the canonical Lie bracket (commutator) of vector fields. (Notice that vector fields with constant coefficients have zero commutator.) One can recognize in (2.9) an analog of Cartan's formulas, see Remark 1.2 .

EXAmPLe 2.2. For the general linear algebra $\mathfrak{g l}(n)$ we have the field

$$
\hat{Q}=-\sum \xi^{i k} \xi^{k j} \frac{\partial}{\partial \xi^{i j}} .
$$

It is tangent to linear submanifolds that correspond to Lie subalgebras in $\mathfrak{g l}(n)$. Same formula works in the super case.

In a more conventional language, the vector field $\hat{Q}$ on $\Pi \mathfrak{g}$ coincides with the Chevalley-Eilenberg differential in $C^{*}(\mathfrak{g})=C^{*}(\mathfrak{g} ; \mathbb{R})$, for ordinary Lie algebras. For Lie superalgebras this can be taken as a convenient definition.

EXAmple 2.3. Consider a general odd (formal) vector field on $\mathbb{R}^{n \mid m}$ :

$$
\hat{Q}=\left(Q_{0}^{k}+\xi^{i} Q_{i}^{k}+\frac{1}{2} \xi^{j} \xi^{i} Q_{i j}^{k}+\frac{1}{3 !} \xi^{l} \xi^{j} \xi^{i} Q_{i j l}^{k}+\ldots\right) \frac{\partial}{\partial \xi^{k}} .
$$

The coefficients $Q_{0}^{k}, Q_{i}^{k}, Q_{i j}^{k}, Q_{i j l}^{k}, \ldots$ define a sequence of $N$-ary operations $(N=$ $0,1,2,3, \ldots)$ on the vector space $\mathbb{R}^{m \mid n}$, and the condition $\hat{Q}^{2}=0$ expands to a linked sequence of "Jacobi identities". If only the quadratic term in the Maclaurin expansion (2.11) is present, we return to the case of a Lie algebra. The general case is a strong homotopy Lie algebra ( $L_{\infty}$-algebra) (due to Stasheff, see [13]).

EXAMPLE 2.4. If instead of commutative algebras of functions on $\mathbb{R}^{n \mid m}$ one considers free noncommutative associative algebras and their odd derivations of square zero (analog of homological fields), then a homotopy version of associative algebras (so called $A_{\infty}$-algebras) will be obtained [24. Again, the purely quadratic case reproduces ordinary (associative) algebras.

Now we can say what is, in this language, a general "bi-" structure for a Lie algebra. Consider the above tetrahedron of vector spaces. A "bi-" structure for $\mathfrak{g}$ is exactly a Lie algebra structure for two of its vertices, with a certain compatibility condition. In view of Theorem 2.3, up to renaming, the list of the corresponding geometric structures is exhausted by three cases:

\begin{tabular}{|r|l|l|l|}
\hline I. & $\mathfrak{g}, \mathfrak{g}^{*}$ Lie algebras & $\begin{array}{l}\text { On } \Pi \mathfrak{g}\left(\text { or } \Pi \mathfrak{g}^{*}\right) \text { there are homological } \\
\text { field } \hat{Q} \text { and odd bracket. }\end{array}$ & $Q S$-manifold \\
\hline II. & $\mathfrak{g}, \Pi \mathfrak{g}^{*}$ Lie algebras & $\begin{array}{l}\text { On } \mathfrak{g}^{*}(\text { or } \Pi \mathfrak{g}) \text { there are homological } \\
\text { field } \hat{Q} \text { and even bracket. }\end{array}$ & $Q P$-manifold \\
\hline III. & $\mathfrak{g}, \Pi \mathfrak{g}$ Lie algebras & $\begin{array}{l}\text { On } \mathfrak{g}^{*}\left(\text { or } \Pi \mathfrak{g}^{*}\right) \text { there are even and odd } \\
\text { brackets. }\end{array}$ & $P S$-manifold \\
\hline
\end{tabular}


One should explain the compatibility conditions. For a pair consisting of a Schouten or Poisson bracket and a homological vector field there is only one reasonable condition, that the field $\hat{Q}$ should be a derivation of the bracket. We call Poisson (Schouten) manifolds endowed with a homological field which is a derivation of the bracket $Q P$-manifolds ( $Q S$-manifolds, respectively).

THEOREM 2.4. A QS-manifold structure on $\Pi \mathfrak{g}$ with a linear Schouten bracket and a quadratic field $\hat{Q}$ is equivalent to a Lie bialgebra structure for $\mathfrak{g}$.

ProOF. As noted above, the vector field $\hat{Q}$ corresponds to the ChevalleyEilenberg differential for the trivial representation. The Lie derivative $L_{\hat{Q}}$ corresponds to the Chevalley-Eilenberg differential for other (nontrivial) representations. Thus the condition that the cobracket on $\mathfrak{g}$ is a cocycle is equivalent to $L_{\hat{Q}} S=0$, for the Hamiltonian $S$ that defines the corresponding Lie-Schouten structure on $\Pi \mathfrak{g}$. On the other hand, we have, by a straightforward calculation,

$$
\hat{Q}\{f, g\}_{S}=\{\hat{Q} f, g\}_{S}+(-1)^{\tilde{f}+1}\{f, \hat{Q} g\}_{S}+(-1)^{\tilde{f}}\left\{f,\left\{L_{\hat{Q}} S, g\right\}\right\},
$$

for the bracket $\{,\}_{S}$ defined by $S$. Hence, if $L_{\hat{Q}} S=0$, then the derivation property holds. Conversely, if the derivation property holds, then for all $f, g$ $\left\{f,\left\{L_{\hat{Q}} S, g\right\}\right\}=0$, and this implies $L_{\hat{Q}} S=0$ by the virtue of the non-degeneracy of the canonical Poisson bracket.

In particular, this is the way to get the correct formulas like $(2.2)$ in the super case $\mathfrak{g}=\mathfrak{g}_{0} \oplus \mathfrak{g}_{1}$. Practically, this theorem can be used instead of the definition in this case.

What about the $Q P$-manifolds? They correspond to an "odd version" of the Lie bialgebra notion.

Definition 2.2. An odd Lie bialgebra is a Lie superalgebra $\mathfrak{g}$ such that the space $\Pi \mathfrak{g}^{*}$ is also a Lie superalgebra so that on the antialgebra $\Pi \mathfrak{g}$ is induced a $Q P$-structure (with linear Poisson bracket and quadratic homological field).

We shall elaborate this in Section 6 .

REMARK 2.1. It should be emphasized that while a Lie algebra $\mathfrak{g}$ can be viewed via three geometric manifestations, listed in Theorem 2.3 , for a "bi-" case there is just one geometric picture. A $Q S$-structure with linear bracket and quadratic field (realized, equivalently, either on $\Pi \mathfrak{g}$ or on $\Pi \mathfrak{g}^{*}$ ) corresponds to a Drinfeld's Lie bialgebra, and a $Q P$-structure with linear bracket and quadratic field (realized, equivalently, either on $\Pi \mathfrak{g}$ or on $\mathfrak{g}^{*}$ ) corresponds to an odd bialgebra.

We reserve the name $P S$-manifold for a manifold with a pair of brackets of opposite parity. The precise compatibility condition in this case is not obvious. We refrain from discussing it here. Geometry of spaces with even and odd bracket was studied, in particular, in [7. For $\mathfrak{g} \oplus \Pi \mathfrak{g}$, an odd endomorphism replaces the pairing.

Now, we need the description of Drinfeld's double in this language. Start from the space $\Pi \mathfrak{g}$. Notice that $\Pi\left(\mathfrak{g} \oplus \mathfrak{g}^{*}\right) \cong T^{*} \Pi \mathfrak{g}$. The natural (symmetric) inner product on $\mathfrak{g} \oplus \mathfrak{g}^{*}$ corresponds to the canonical symplectic structure on $T^{*} \Pi \mathfrak{g}$. Denote coordinates on $\Pi \mathfrak{g}$ by $\xi^{i}$ and their conjugate momenta by $\xi_{j}$. As we know from Section 1, a linear Schouten bracket on $\Pi \mathfrak{g}$ is specified by an odd Hamiltonian of the form $S=(1 / 2) \xi^{l} S_{l}^{n m} \xi_{m} \xi_{n} \in C^{\infty}\left(T^{*} \Pi \mathfrak{g}\right)$. On the other hand, a vector field $\hat{Q}=(1 / 2) \xi^{j} \xi^{i} Q_{i j}^{k} \partial / \partial \xi^{k} \in \operatorname{Vect}(\Pi \mathfrak{g})$ induces the Hamiltonian $Q=(1 / 2) \xi^{j} \xi^{i} Q_{i j}^{k} \xi_{k} \in$ $C^{\infty}\left(T^{*} \Pi \mathfrak{g}\right)$. 
THEOREM 2.5. The following statements hold for $T^{*} \Pi \mathfrak{g} \cong \Pi\left(\mathfrak{g} \oplus \mathfrak{g}^{*}\right)$ :

1. The QS-condition for $\Pi \mathfrak{g}$ with the field $\hat{Q}$ and the Schouten tensor $S$ is equivalent to the vanishing of the Poisson bracket

$$
\{Q, S\}=\frac{1}{4}\left\{\xi^{j} \xi^{i} Q_{i j}^{k} \xi_{k}, \xi^{l} S_{l}^{n m} \xi_{m} \xi_{n}\right\}=0 .
$$

2. The function $=Q+S$ on $T^{*} \Pi \mathfrak{g}$ satisfies $\{Q+S, Q+S\}=0$, so the Hamiltonian vector field $\hat{Q}_{D}:=X_{Q+S} \in \operatorname{Vect}\left(T^{*} \Pi \mathfrak{g}\right)$ is homological. It corresponds to the Lie algebra structure in Drinfeld's double $\mathfrak{d}=\mathfrak{g} \oplus \mathfrak{g}^{*}$.

Proof. To prove part 1 , notice that $\{Q, S\}=L_{\hat{Q}} S$, thus the equation $\{Q, S\}=$ 0 is equivalent to the $Q S$-condition (see the proof of Theorem 2.4). To prove part 2, notice that the equation $\{Q+S, Q+S\}=0$ implies the equation $\hat{Q}_{D}^{2}=X_{Q+S}^{2}=0$ for the corresponding Hamiltonian field. Finally, to show that $\hat{Q}_{D}$ gives exactly the Lie bracket in Drinfeld's double, we compare the explicit formulas. For $X_{Q}$ we obtain, using the general formula (1.16):

$$
\begin{aligned}
X_{Q}=\{Q,\}= & (-1)^{(\tilde{k}+1)(\tilde{Q}+1)} \frac{\partial Q}{\partial \xi_{k}} \frac{\partial}{\partial \xi^{k}}-(-1)^{(\tilde{k}+1) \tilde{Q}} \frac{\partial Q}{\partial \xi^{k}} \frac{\partial}{\partial \xi_{k}} \\
& =\frac{\partial Q}{\partial \xi_{k}} \frac{\partial}{\partial \xi^{k}}+(-1)^{\tilde{k}} \frac{\partial Q}{\partial \xi^{k}} \frac{\partial}{\partial \xi_{k}}=\frac{1}{2} \xi^{j} \xi^{i} Q_{i j}^{k} \frac{\partial}{\partial \xi^{k}}+(-1)^{\tilde{k}} \xi^{i} Q_{i k}^{j} \xi_{j} \frac{\partial}{\partial \xi_{k}}
\end{aligned}
$$

Similarly, we obtain

$$
\begin{aligned}
X_{S}=\{S,\}=\frac{\partial S}{\partial \xi_{k}} \frac{\partial}{\partial \xi^{k}}+(-1)^{\tilde{k}} \frac{\partial S}{\partial \xi^{k}} \frac{\partial}{\partial \xi_{k}} & \\
& =\xi_{n} \xi^{l} S_{l}^{n k} \frac{\partial}{\partial \xi^{k}}+(-1)^{\tilde{k}} \frac{1}{2} S_{k}^{n m} \xi_{m} \xi_{n} \frac{\partial}{\partial \xi_{k}}
\end{aligned}
$$

Thus $\hat{Q}_{D}=X_{Q}+X_{S}$ reads

$$
\hat{Q}_{D}=\left(\frac{1}{2} \xi^{j} \xi^{i} Q_{i j}^{k}+\xi_{j} \xi^{i} S_{i}^{j k}\right) \frac{\partial}{\partial \xi^{k}}+(-1)^{\tilde{k}}\left(\frac{1}{2} S_{k}^{i j} \xi_{j} \xi_{i}+\xi^{i} Q_{i k}^{j} \xi_{j}\right) \frac{\partial}{\partial \xi_{k}},
$$

and in the case of a purely even $\mathfrak{g}$ it is reduced to

$$
\left(\frac{1}{2} \xi^{j} \xi^{i} Q_{i j}^{k}+\xi_{j} \xi^{i} S_{i}^{j k}\right) \frac{\partial}{\partial \xi^{k}}+\left(\frac{1}{2} S_{k}^{i j} \xi_{j} \xi_{i}-\xi_{j} \xi^{i} Q_{i k}^{j}\right) \frac{\partial}{\partial \xi_{k}},
$$

which reproduces formulas $(2.3 \sqrt{2.5})$ for the Lie bracket.

REMARK 2.2. It should be noted that the description of the double of a Lie bialgebra in terms of a pair of commuting Hamiltonians was first done in 14. A similar description for Lie "quasi-bialgebras" was obtained in [9].

Theorem 2.5 gives for $T^{*} \Pi \mathfrak{g}$ a $Q$-structure corresponding to the Lie bracket in the double. To get on $T^{*} \Pi \mathfrak{g}$ an $S$-structure corresponding to the cobracket, consider the cotangent bundle of $T^{*} \Pi \mathfrak{g}$. Denote by $\pi_{i}, \pi^{i}$ the conjugate momenta for $\xi^{i}, \xi_{i}$, respectively. Notice that $\pi_{i}$ transforms as $\xi_{i}$, and $\pi^{i}$ as $(-1)^{\tilde{\tau}} \xi^{i}$ (see a more general statement in Section 3). Hence $r=-(-1)^{\tilde{i}} \pi^{i} \pi_{i}=\pi_{i} \pi^{i}$ is an invariantly defined function on $T^{*} T^{*} \Pi \mathfrak{g}$. Consider the following linear map $C^{\infty}\left(T^{*} \Pi \mathfrak{g}\right) \rightarrow$ $C^{\infty}\left(T^{*} T^{*} \Pi \mathfrak{g}\right), f \mapsto \bar{f}:=\left\{p_{X_{f}}, r\right\}$. 
Lemma 2.1. For an arbitrary function $f \in C^{\infty}\left(T^{*} \Pi \mathfrak{g}\right)$ there is an explicit formula

$$
\bar{f}=\left\{p_{X_{f}}, r\right\}=(-1)^{(\tilde{\imath}+\tilde{\jmath}) \tilde{f}} \frac{\partial^{2} f}{\partial \xi^{j} \partial \xi^{i}} \pi^{i} \pi^{j}-(-1)^{(\tilde{\imath}+\tilde{\jmath})(\tilde{f}+1)} \frac{\partial^{2} f}{\partial \xi_{j} \partial \xi_{i}} \pi_{i} \pi_{j} .
$$

Proof. Notice, first, that the bracket of an arbitrary $g \in C^{\infty}\left(T^{*} T^{*} \Pi \mathfrak{g}\right)$ with $r=\pi_{i} \pi^{i}$ equals

$$
\{g, r\}=-(-1)^{(\tilde{\jmath}+1) \tilde{g}} \frac{\partial g}{\partial \xi^{j}} \pi^{j}-(-1)^{(\tilde{\jmath}+1)(\tilde{g}+1)} \frac{\partial g}{\partial \xi_{j}} \pi_{j} .
$$

Now, for an arbitrary $f \in C^{\infty}\left(T^{*} \Pi \mathfrak{g}\right)$ we get the function

$$
p_{X_{f}}=(-1)^{(\tilde{\imath}+1)(\tilde{f}+1)} \frac{\partial f}{\partial \xi_{i}} \pi_{i}-(-1)^{(\tilde{\imath}+1)} \tilde{f} \frac{\partial f}{\partial \xi^{i}} \pi^{i}
$$

on $T^{*} T^{*} \Pi \mathfrak{g}$. Substituting $(2.17)$ into $(2.16)$, we obtain formula $(2.15)$ by a direct simplification (which we omit). Notice that the terms containing "cross" products like $\pi_{i} \pi^{j}$ remarkably cancel.

Theorem 2.6. Consider $S_{D}:=(1 / 2)\left\{Q_{D}, r\right\} \in C^{\infty}\left(T^{*} T^{*} \Pi \mathfrak{g}\right)$, where $Q_{D} \in$ $C^{\infty}\left(T^{*} T^{*} \Pi \mathfrak{g}\right)$ corresponds to the vector field $\hat{Q}_{D}$. Then $S_{D}$ is a Schouten tensor for $T^{*} \Pi \mathfrak{g}$, and together with the field $\hat{Q}_{D}$ it gives a $Q S$-structure on $T^{*} \Pi \mathfrak{g}$. The Schouten bracket specified by $S_{D}$ corresponds to the cobracket in Drinfeld's double $\mathfrak{d}=\mathfrak{g} \oplus \mathfrak{g}^{*}$.

Proof. We have $S_{D}=(1 / 2)(\overline{Q+S})=(1 / 2)(\bar{Q}+\bar{S})$, in the notation of Lemma 2.1. Applying the lemma to the functions $Q$ and $S$, we obtain the formulas

$$
\begin{aligned}
\bar{Q} & =(-1)^{\tilde{\imath}+\tilde{\jmath}} Q_{i j}^{k} \xi_{k} \pi^{j} \pi^{i}=(-1)^{\tilde{\jmath}} \pi^{j}(-1)^{\tilde{\imath}} \pi^{i} Q_{i j}^{k} \xi_{k} \\
\bar{S} & =-\xi^{k} S_{k}^{i j} \pi_{j} \pi_{i} .
\end{aligned}
$$

Here in $(2.18)$ we changed the order and separated the factors $(-1)^{\tau} \pi^{i}$. The point is that the correspondence $\xi^{i} \leftrightarrow(-1)^{\tilde{\imath}} \pi^{i}$ respects the Poisson bracket: $\left\{\xi_{i}, \xi^{j}\right\}_{T^{*} \Pi \mathfrak{g}}=$ $\delta_{i}^{j}=\left\{\xi_{i},(-1)^{\tilde{j}} \pi^{j}\right\}_{T^{*} T^{*} \Pi \mathfrak{g}}$. In the same way, $\left\{\xi_{i}, \xi^{j}\right\}_{T^{*} \Pi \mathfrak{g}}=\delta_{i}^{j}=\left\{\pi_{i}, \xi^{j}\right\}_{T^{*} T^{*} \Pi \mathfrak{g}}$. Hence $\{\bar{Q}, \bar{Q}\}=4\{Q, Q\}=0,\{\bar{S}, \bar{S}\}=4\{S, S\}=0$ by the virtue of these correspondences, and $\{\bar{Q}, \bar{S}\}=0$ because $\bar{Q}$ and $\bar{S}$ do not contain conjugate variables. Thus, $\left\{S_{D}, S_{D}\right\}=0$. The identity $\left\{Q_{D}, S_{D}\right\}=0$ holds by the construction of $S_{D}$. To notice that $S_{D}$ corresponds to the cobracket in Drinfeld's double (up to a common sign), it suffices to compare the explicit formula obtained

$$
S_{D}=\frac{1}{2}(-1)^{\tilde{\imath}+\tilde{\jmath}} Q_{i j}^{k} \xi_{k} \pi^{j} \pi^{i}-\frac{1}{2} \xi^{k} S_{k}^{i j} \pi_{j} \pi_{i}
$$

with formulas $(2.62 .7)$ in the even case.

(It is clear now how to get an "odd analog" of Drinfeld's double. Let $\mathfrak{g}$ be an odd bialgebra, so $\Pi \mathfrak{g}$ is a $Q P$-manifold with the quadratic $\hat{Q}$ and linear bracket. Instead of $T^{*} \Pi \mathfrak{g}$ we consider the supermanifold $\Pi T^{*} \Pi \mathfrak{g} \cong \Pi \mathfrak{g} \oplus \mathfrak{g}^{*}$. In a way similar to the above, it can be proved that $\Pi T^{*} \Pi \mathfrak{g}$ has a desired $Q P$-structure, corresponding to an odd bialgebra structure in $\mathfrak{g} \oplus \Pi \mathfrak{g}^{*}$. The space $\mathfrak{g} \oplus \Pi \mathfrak{g}^{*}$ with this structure will be called the odd double of an odd bialgebra $\mathfrak{g}$. We will return to this construction in Section 6 and give details.) 
REMARK 2.3. Drinfeld's classical double is, by the construction, a coboundary Lie bialgebra (see [3] ). That means that $S_{D}=\left\{Q_{D}, r / 2\right\}$, in our language. Suppose that for an arbitrary Lie algebra $\mathfrak{g}$ an even Hamiltonian $r \in C^{\infty}\left(T^{*} \Pi \mathfrak{g}\right)$ of the form $r=r^{i j} \pi_{i} \pi_{j}$ (with constant $r^{i j}$ ) is considered. The Poisson bracket with the odd Hamiltonian $Q=(1 / 2) \xi^{j} \xi^{i} Q_{i j}^{k} \pi_{k}$ corresponding to the Lie algebra structure, $\{Q, r / 2\}=: S$, is quadratic in $\pi_{i}: S=(1 / 2) \xi^{i} S_{i}^{j k} \pi_{k} \pi_{j}$. The linear odd bracket on $\Pi \mathfrak{g}$ defined as $\{f, g\}_{S}=\{f,\{S, g\}\}$ is automatically compatible with $Q$. It satisfies the Jacobi identity (thus making $\mathfrak{g}$ into a Lie bialgebra) if and only if the Poisson bracket $\{\{Q, r\},\{Q, r\}\}$ vanishes. Since $\{Q,\{r,\{Q, r\}\}\}=\{\{Q, r\},\{Q, r\}\}$, this is equivalent to

$$
\left\{Q,\{r, r\}_{Q}\right\}=0
$$

where the inner bracket denotes the operation

$$
r_{1}, r_{2} \mapsto\left\{r_{1}, r_{2}\right\}_{Q}:=\left\{r_{1},\left\{Q, r_{2}\right\}\right\}=\left\{\left\{r_{1}, Q\right\}, r_{2}\right\}
$$

on Hamiltonians $r_{1}, r_{2}$ depending only on $\pi_{i}$ (this is exactly the Lie-Schouten bracket on $\left.\Pi \mathfrak{g}^{*}\right)$. The equation $(\overline{2.21})$ is satisfied if, in particular,

$$
\{r, r\}_{Q}=0 \text {. }
$$

This is the classical Yang-Baxter equation for $r$, while (2.21) is the "generalized" classical Yang-Baxter equation. Notice that $r=\pi_{i} \pi^{i}$ for Drinfeld's double satisfies $(2.21)$, but it does not satisfy $(2.23),\{r, r\}_{Q_{D}}$ being a certain canonical $\hat{Q}_{D^{-}}$ invariant cubic expression in $\pi_{i}, \pi^{j}$ (in the standard language $r$ is said to be a solution of the "modified" classical Yang-Baxter equation).

\section{Doubles for Lie bialgebroids}

In this section we review the results of D. Roytenberg 22 and A. Vaintrob 26, who independently applied super methods to Lie bialgebroids. As noted before, we give a "superized" version, i.e., consider from the beginning super Lie (bi)algebroids over supermanifolds. Lie bialgebroids were originally introduced by Mackenzie and $\mathrm{Xu}$ [17. Important results, which particularly nicely fit into the super framework (but were originally formulated without it), are due to Y. KosmannSchwarzbach [10].

Consider a Lie algebroid $E \rightarrow M$ as defined in Section 1, In direct analogy with the tangent bundles and Lie algebras, it gives rise to a differential algebra, which is $C^{\infty}(\Pi E)$ with the homological vector field $\hat{Q}=\hat{Q}_{E} \in \operatorname{Vect}(\Pi E)$,

$$
\hat{Q}=\xi^{i} Q_{i}^{a}(x) \frac{\partial}{\partial x^{a}}+\frac{1}{2} \xi^{j} \xi^{i} Q_{i j}^{k}(x) \frac{\partial}{\partial \xi^{k}} .
$$

(Here we use the notation $x^{a}$ for coordinates in the base and $\xi^{i}$ for coordinates in the fiber of $\Pi E$.) In the purely even case, $C^{\infty}(\Pi E)$ is the algebra of smooth sections of the exterior bundle $C^{\infty}\left(M, \Lambda\left(E^{*}\right)\right)$. The differential $d_{E}=\hat{Q}_{E}: C^{\infty}\left(M, \Lambda\left(E^{*}\right)\right) \rightarrow$ $C^{\infty}\left(M, \Lambda\left(E^{*}\right)\right)$ is analogous to the de Rham and Chevalley-Eilenberg differentials (which are both due to Cartan). The coefficients $Q_{i j}^{k}(x)$ describe the Lie brackets of a local basis of sections in $E$, while $Q_{i}^{a}(x)$ correspond to the anchor map $a: C^{\infty}(M, E) \rightarrow \operatorname{Vect}(M)$. The bracket $[,]_{E}$ and the anchor are recovered from 
$\hat{Q}$ by the formulas similar to $(2.9)$ :

$$
\begin{aligned}
a(u) f & =(-1)^{\tilde{u}}\left[i_{u},[\hat{Q}, f]\right]=(-1)^{\tilde{u}}\left[\left[i_{u}, \hat{Q}\right], f\right] \\
i_{[u, v]_{E}} & =\left[i_{u},\left[\hat{Q}, i_{v}\right]\right]=\left[\left[i_{u}, \hat{Q}\right], i_{v}\right]
\end{aligned}
$$

where $i_{u}=(-1)^{\tilde{u}} u^{i}(x) \partial / \partial \xi^{i}$ for $u=u^{i}(x) e_{i} \in C^{\infty}(M, E)$. In particular, for the basis sections we have

$$
a\left(e_{i}\right)=Q_{i}^{a}(x) \frac{\partial}{\partial x^{a}}, \quad\left[e_{i}, e_{j}\right]_{E}=(-1)^{\tilde{j}} Q_{i j}^{k}(x) e_{k} .
$$

The identity $\hat{Q}^{2}=0$ compactly contains all the properties of the anchor and the bracket. Thus, we can take it as an equivalent definition of a Lie algebroid structure. A vector bundle endowed with $\hat{Q}$ of the form (3.1) such that $\hat{Q}^{2}=0$ is, by definition, a Lie antialgebroid. $\Pi E \rightarrow M$ is the Lie antialgebroid corresponding to the Lie algebroid $E \rightarrow M$.

The Lie algebroid structure in $E$ also defines a Schouten bracket in the algebra $C^{\infty}\left(\Pi E^{*}\right)$, which in the purely even case is the algebra of smooth sections $C^{\infty}(M, \Lambda(E))$, and a Poisson bracket in the algebra $C^{\infty}\left(E^{*}\right)$. Thus, as for Lie algebras, there are a $Q$-manifold, an $S$-manifold and a $P$-manifold associated with a Lie algebroid.

Return for a moment to a purely even situation. Let the dual bundle $E^{*} \rightarrow M$ also have a structure of a Lie algebroid. Then, there is a differential

$$
d_{E^{*}}: C^{\infty}(M, \Lambda(E)) \rightarrow C^{\infty}(M, \Lambda(E)) .
$$

It can be applied, in particular, to sections of $E \rightarrow M$ considered as sections of the exterior bundle.

Definition 3.1 (Mackenzie-Xu 17]). A Lie bialgebroid over a manifold $M$ is a Lie algebroid $E \rightarrow M$ for which the dual bundle $E^{*} \rightarrow M$ is also a Lie algebroid so that the differential $d_{E^{*}}$ satisfies the derivation property for the bracket of sections of $E$.

It was shown by Y. Kosmann-Schwarzbach 10 that it is equivalent and more convenient to require that $d_{E^{*}}$ is a derivation of the Schouten bracket in the whole algebra $C^{\infty}(M, \Lambda(E))$. It can also be shown (though it is not straightforward at this point) that $E$ and $E^{*}$ here can be interchanged. Assuming this, the definition can be put into a super language (allowing to cover the general case) as follows:

Definition 3.2 (equivalent). A (super) Lie bialgebroid over a supermanifold $M$ is a vector bundle $E \rightarrow M$ such that the space of the opposite vector bundle $\Pi E \rightarrow M$ is a $Q S$-manifold, with the homological field of the form

$$
\hat{Q}=\xi^{i} Q_{i}^{a}(x) \frac{\partial}{\partial x^{a}}+\frac{1}{2} \xi^{j} \xi^{i} Q_{i j}^{k}(x) \frac{\partial}{\partial \xi^{k}}
$$

and the non-vanishing Schouten brackets of coordinates of the form

$$
\left\{\xi^{i}, x^{a}\right\}_{S}=(-1)^{\tilde{\imath}} S^{i a}(x), \quad\left\{\xi^{i}, \xi^{j}\right\}_{S}=(-1)^{\tilde{\imath}} \xi^{k} S_{k}^{i j}(x) .
$$

That means that the bracket is given by the Hamiltonian $S=S_{E} \in C^{\infty}\left(T^{*} \Pi E\right)$,

$$
S=S^{i a}(x) p_{a} \pi_{i}+\frac{1}{2} \xi^{k} S_{k}^{i j}(x) \pi_{j} \pi_{i} .
$$


THEOREM 3.1. E is a Lie bialgebroid if and only if the Poisson bracket $\{Q, S\}$ vanishes, where $Q=p(\hat{Q}) \in C^{\infty}\left(T^{*} \Pi E\right)$ is the Hamiltonian corresponding to the vector field $\hat{Q}$.

Proof. Similarly to Theorem 2.4 the condition of a Lie bialgebroid is equivalent to $L_{\hat{Q}_{E}} S_{E}=0$, and $L_{\hat{Q}_{E}} S_{E}=\{Q, S\}$.

This definition is not manifestly symmetric with respect to $E$ and $E^{*}$. A symmetric description can be achieved with the help of the following theorem (see Mackenzie and $\mathrm{Xu}$ [17; we provide a proof in the Appendix).

THEOREM 3.2. For an arbitrary vector bundle $E \rightarrow M$ there is a natural diffeomorphism of the cotangent bundles $F: T^{*} E \cong T^{*} E^{*}$, which preserves the symplectic structure. In coordinates:

$$
F:\left(x^{a}, y^{i}, p_{a}, p_{i}\right) \mapsto\left(x^{a}, y_{i}, p_{a}, p^{i}\right)=\left(x^{a}, p_{i}, p_{a},-(-1)^{\tilde{\imath}} y^{i}\right) .
$$

Here $y^{i}$ stand for (left) coordinates in the fiber of $E, y_{i}$ stand for the contragredient right coordinates in the fiber of $E^{*}$, and $p_{i}, p^{i}$ for the respective conjugate momenta.

Consider a Lie algebroid $\Pi E$. Let the vector field $\hat{Q}=\hat{Q}_{E} \in \operatorname{Vect}(\Pi E)$ be given by (3.5). The corresponding Hamiltonian $Q_{E} \in C^{\infty}\left(T^{*} \Pi E\right)$ is

$$
Q_{E}=\xi^{i} Q_{i}^{a}(x) p_{a}+\frac{1}{2} \xi^{j} \xi^{i} Q_{i j}^{k}(x) \pi_{k} .
$$

We shall apply Theorem 3.2 to the vector bundle $\Pi E$. Suppose $E^{*}$ is also a Lie algebroid. Let $\hat{Q}_{E^{*}} \in \operatorname{Vect}\left(\Pi E^{*}\right)$ be the homological field defining the algebroid structure in $E^{*}$ and the corresponding Hamiltonian $Q_{E^{*}} \in C^{\infty}\left(T^{*} \Pi E^{*}\right)$ be

$$
Q_{E^{*}}=\xi_{i} Q^{i a}(x) p_{a}+\frac{1}{2} \xi_{j} \xi_{i} Q_{k}^{i j}(x) \pi^{k}
$$

Here $\xi_{i}$ with lower indices denote the coordinates in the fiber of $\Pi E^{*}$ and $\pi^{i}$ the conjugate momenta. In particular, the anchor and bracket in $E^{*}$ will be given by

$$
a\left(e^{i}\right)=Q^{i a}(x) \frac{\partial}{\partial x^{a}}, \quad\left[e^{i}, e^{j}\right]_{E^{*}}=(-1)^{\tilde{\jmath}} Q_{k}^{i j}(x) e^{k} .
$$

By Theorem 3.2 , we identify $T^{*} \Pi E$ and $T^{*} \Pi E^{*}$. Rewritten in coordinates $\xi^{i}=$ $(-1)^{\tilde{\imath}} \pi^{i}, \pi_{i}=\xi_{i}$, the Hamiltonian $Q_{E^{*}}$ becomes

$$
\begin{aligned}
& Q_{E^{*}}=\pi_{i} Q^{i a}(x) p_{a}+\frac{1}{2} \pi_{j} \pi_{i} Q_{k}^{i j}(x)(-1)^{\tilde{k}} \xi^{k}= \\
& Q^{i a}(x) p_{a} \pi_{i}+\frac{1}{2}(-1)^{\tilde{k}} Q_{k}^{i j}(x) \xi^{k} \pi_{j} \pi_{i} .
\end{aligned}
$$

TheOrem 3.3 (Roytenberg [22, Vaintrob [26]). The Hamiltonians $S_{E}$ and $Q_{E^{*}}$ coincide. Hence, $E$ is a Lie bialgebroid if and only if the Hamiltonians $Q_{E}, Q_{E^{*}} \in C^{\infty}\left(T^{*} \Pi E\right)=C^{\infty}\left(T^{*} \Pi E^{*}\right)$ commute.

Proof. The key statement is that $Q_{E^{*}}=S_{E}$ (after the identification of $T^{*} \Pi E$ and $\left.T^{*} \Pi E^{*}\right)$. The second statement would follow from Theorem 3.1. We have to show that the Schouten bracket on $\Pi E$ induced by the algebroid structure in $E^{*}$ and specified by $S_{E}$ is the same as given by the quadratic Hamiltonian (3.11). To this end, we check the non-vanishing Schouten brackets for coordinates. We get $\left\{\xi^{i}, x^{a}\right\}_{S}=\left\{\theta\left(e^{i}\right), x^{a}\right\}_{S}=(-1)^{\tilde{\imath}} a\left(e^{i}\right) x^{a}=(-1)^{\tilde{\tau}} Q^{i a}$ and $\left\{\xi^{i}, \xi^{j}\right\}_{S}=\theta\left(\left[e^{i}, e^{j}\right]_{E^{*}}\right)=$ $\theta\left((-1)^{\tilde{\jmath}} Q_{k}^{i j} e^{k}\right)=(-1)^{\tilde{\imath}+\tilde{k}} Q_{k}^{i j} \xi^{k}$, by the definition of the Schouten bracket induced 
by an algebroid structure and formulas (3.10). (Here $\theta: C^{\infty}\left(M, E^{*}\right) \rightarrow C^{\infty}(\Pi E)$ is $\alpha=e^{i} \alpha_{i}(x) \mapsto \theta(\alpha)=\xi^{i} \alpha_{i}(x)$.) This exactly coincides with the Schouten brackets given by the Hamiltonian (3.11). Hence, $S_{E}=Q_{E^{*}}$.

It follows that the condition of a Lie bialgebroid is self-dual. Under the identification $T^{*} \Pi E=T^{*} \Pi E^{*}$ the $Q S$-structure on $\Pi E$ corresponds to the $Q S$-structure on $\Pi E^{*}$ so that the homological field and the bracket effectively exchange places. Note, however, that this self-duality is an extra statement not necessary for writing the condition of a Lie bialgebroid in the Poisson form $\{Q, S\}=0$. It does not make sense in a nonlinear case considered in Section 5 .

How to get an analog of Drinfeld's double for a bialgebroid E? Roytenberg suggested the following argument. Consider the Hamiltonian vector fields on the supermanifold $T^{*} \Pi E$ corresponding to the Hamiltonians $Q=Q_{E}$ and $S=Q_{E^{*}}$. According to the general formulas, for an odd function on $T^{*} \Pi E$ we have

$$
X_{f}=\{f,\}=\frac{\partial f}{\partial p_{a}} \frac{\partial}{\partial x^{a}}-(-1)^{\tilde{a}} \frac{\partial f}{\partial x^{a}} \frac{\partial}{\partial p_{a}}+\frac{\partial f}{\partial \pi_{i}} \frac{\partial}{\partial \xi^{i}}+(-1)^{\tilde{\imath}} \frac{\partial f}{\partial \xi^{i}} \frac{\partial}{\partial \pi_{i}} .
$$

Hence, we get:

$$
\begin{aligned}
& X_{Q}=\xi^{i} Q_{i}^{a} \frac{\partial}{\partial x^{a}}-(-1)^{\tilde{a}}\left(\frac{\partial Q_{i}^{b}}{\partial x^{a}} p_{b} \xi_{i}+\frac{1}{2} \frac{\partial Q_{i j}^{k}}{\partial x^{a}} \pi_{k} \xi^{j} \xi^{i}\right) \frac{\partial}{\partial p_{a}} \\
&+\frac{1}{2} \xi^{j} \xi^{i} Q_{i j}^{k} \frac{\partial}{\partial \xi^{k}}+(-1)^{\tilde{k}}\left(Q_{k}^{a} p_{a}+\xi^{j} Q_{j k}^{i} \pi_{i}\right) \frac{\partial}{\partial \pi_{k}} \\
& X_{S}=\pi_{i} Q^{i a} \frac{\partial}{\partial x^{a}}-(-1)^{\tilde{a}}\left(\frac{\partial Q^{i b}}{\partial x^{a}} p_{b} \pi_{i}+\frac{1}{2}(-1)^{\tilde{k}} \frac{\partial Q_{k}^{i j}}{\partial x^{a}} \xi^{k} \pi_{j} \pi_{i}\right) \frac{\partial}{\partial p_{a}} \\
&+\left(Q^{k a} p_{a}+(-1)^{\tilde{\tau}} \pi_{j} Q_{i}^{j k} \xi^{i}\right) \frac{\partial}{\partial \xi^{k}}+(-1)^{\tilde{k}} \frac{1}{2} \pi_{j} \pi_{i} Q_{k}^{i j} \frac{\partial}{\partial \pi_{k}}
\end{aligned}
$$

Mimicking the construction of Drinfeld's double for Lie bialgebras, define $\hat{Q}_{D}:=$ $X_{Q}+X_{S}$, and for the vector field $\hat{Q}_{D}$ on $T^{*} \Pi E$ we get

$$
\begin{gathered}
\hat{Q}_{D}=\left(\xi^{i} Q_{i}^{a}+\pi_{i} Q^{i a}\right) \frac{\partial}{\partial x^{a}} \\
-(-1)^{\tilde{a}}\left(\frac{\partial Q_{i}^{b}}{\partial x^{a}} p_{b} \xi_{i}+\frac{\partial Q^{i b}}{\partial x^{a}} p_{b} \pi_{i}+\frac{1}{2} \frac{\partial Q_{i j}^{k}}{\partial x^{a}} \pi_{k} \xi^{j} \xi^{i}+\frac{1}{2}(-1)^{\tilde{k}} \frac{\partial Q_{k}^{i j}}{\partial x^{a}} \xi^{k} \pi_{j} \pi_{i}\right) \frac{\partial}{\partial p_{a}} \\
+\left(Q^{k a} p_{a}+(-1)^{\tilde{\imath}} \pi_{j} Q_{i}^{j k} \xi^{i}+\frac{1}{2} \xi^{j} \xi^{i} Q_{i j}^{k}\right) \frac{\partial}{\partial \xi^{k}} \\
+(-1)^{\tilde{k}}\left(Q_{k}^{a} p_{a}+\xi^{j} Q_{j k}^{i} \pi_{i}+\frac{1}{2} \pi_{j} \pi_{i} Q_{k}^{i j}\right) \frac{\partial}{\partial \pi_{k}} .
\end{gathered}
$$

Obviously, the field $\hat{Q}_{D}$ is homological, because $Q$ and $S$ commute. Some terms in this lengthy expression are recognizable as similar to those in Drinfeld's double in the bialgebra case (compare with $(2.14)$ ). However, because of the presence of terms cubic in variables $\xi^{i}, \pi_{j}$, the homological field $\hat{Q}_{D}$ cannot be related with a Lie algebroid over $M$.

In Roytenberg's thesis 22 it was suggested to call the field $\hat{Q}_{D} \in \operatorname{Vect}\left(T^{*} \Pi E\right)$ the Drinfeld double of the Lie bialgebroid E. 
It should be once again emphasized that the supermanifold $T^{*} \Pi E$ does not have the form $\Pi A$ for any vector bundle $A \rightarrow M$ and that the field $\hat{Q}_{D}$ contains terms not appropriate for Lie (anti)algebroids over $M$.

Two other constructions were suggested as analogs of Drinfeld's double. Liu, Weinstein and $\mathrm{Xu} 15$ considered the direct sum $E \oplus E^{*}$ as a vector bundle over $M$ and endowed it with a structure of a Courant algebroid, a notion that they defined. Roughly, it is a vector bundle with a bracket of sections which satisfies the Jacobi identity up to certain anomaly expressible in terms of a non-degenerate inner product (see [15], also [22]). There is a natural projection $T^{*} \Pi E \rightarrow \Pi\left(E \oplus E^{*}\right)$. Roytenberg 22 showed that the Courant algebroid structure in $E \oplus E^{*}$ is obtained by exactly the same procedure (described in Section 2 above) that reproduces the Lie bracket on the double of a Lie bialgebra in case the base manifold is a point. K. Mackenzie [19, 20] suggested to consider as a double of a Lie bialgebroid $E$ the manifold $T^{*} E$ together with the structure of a "double Lie algebroid", in a categorical sense precisely defined by him. The easy part of this notion as is follows. There is a diagram

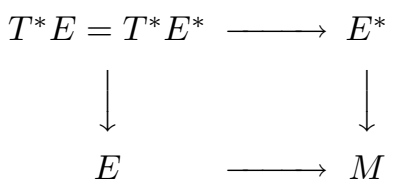

It can be seen that each arrow is a vector bundle projection, and the horizontal arrows constitute a morphism of the vertical bundle structure, and vice versa. Such structure is called a double vector bundle. Moreover, it turns out that there are Lie algebroid structures on all four sides, and, in addition, certain conditions are satisfied 19, 20 which allow to describe the diagram (3.16) as a double Lie algebroid over $M$ (this is the hard part). Again, the "direct sum object" $E \oplus E^{*}$ sits inside this picture, due to the projection $T^{*} E \rightarrow E \oplus E^{*}$. (I hope to elucidate the relation of Mackenzie's beautiful picture with the super approach elsewhere.)

Since there is no hope to interpret the homological field $\hat{Q}_{D}$ in terms of a vector bundle over $M$, other approach should be taken. We think that the key hint is in the following fact. One can check that a change of coordinates in $T^{*} \Pi E$ (see the Appendix) preserves the following $\mathbb{Z}$-gradings: $\# p+\# \pi$ and $\# p+\# \xi$ (the symbol \# means total degree in a given variable), as well as their linear combinations, viz., $\# \xi-\# \pi$ (cf. with the similar gradings considered in $[\mathbf{2 7}, \mathbf{2 8}$.) It was observed in 22 that the vector field $\hat{Q}_{D}$ is homogeneous w.r.t. a grading defined as

$$
(\# p+\# \pi)+(\# p+\# \xi)=2 \# p+\# \pi+\# \xi
$$

of degree +1 . For a vector bundle $E \rightarrow M$, one can uniquely characterize the homological fields on $\Pi E$ of the form (3.1) corresponding to a Lie algebroid structures in $E$ as having the degree +1 in linear fiber coordinates $\xi^{i}$.

Hence, a $\mathbb{Z}$-grading should be regarded as a replacement for an absent linear structure. The trick is that, differently from the vector bundle case, one should allow to count degrees in different variables with possibly different weights (a situation well known in algebraic topology). We systematically develop this approach in the next sections. 


\section{Graded manifolds}

Definition 4.1. Consider a domain $U \subset \mathbb{R}^{n \mid m}$ with coordinates $x^{a}$ (some even, some odd). Assign to them weights: $w\left(x^{a}\right)=w_{a} \in \mathbb{Z}$. Consider the algebra of all smooth functions of $x^{a}$ that are polynomial in coordinates of nonzero weight. Denote it $A$. It is a $\mathbb{Z}$-graded algebra (as well as $\mathbb{Z}_{2}$-graded; these two gradings are independent). We assume that $U$ is cylindrical in the directions of even variables of nonzero weight. There are naturally defined morphisms of pairs $(U, A)$ (homomorphisms of algebras in particular must respect weight). A graded manifold is a locally ringed space modeled on such pairs. Morphisms of graded manifolds preserve weight of functions.

Simply speaking, a graded manifold $M$ is a supermanifold with a privileged class of atlases in which particular coordinates are assigned numbers called "weight" so that the changes of coordinates respect these weights.

On every graded manifold there is an action of the scaling transformations (group $\mathbb{R}^{*}$ under multiplication). In coordinates, $\left(x^{a}\right) \mapsto\left(\lambda^{w_{a}} x^{a}\right), \lambda \in \mathbb{R}^{*}$.

REMARK 4.1. It is possible to consider non-integer weights as well. The difference between coordinates of zero and nonzero weight is similar to the distinction between physical quantities that in some system of units are dimensionless and those that are not (e.g., measured in centimeters). It is possible to consider a function like sine or logarithm of a dimensionless quantity, but not of the one that is measured in centimeters or grams, for which only homogeneous algebraic operations are permitted.

The idea of a "graded manifold" is, of course, very natural. Formal "Z्Z-graded manifolds" were used by Kontsevich in $\left[\mathbf{8}\right.$, however, his $\mathbb{Z}$-grading simply underlies $\mathbb{Z}_{2}$-grading. Our definition is very close to the notion of " $N$-manifolds" suggested by Ševera in $\mathbf{3 3}$. However, Ševera also couples $\mathbb{Z}_{2}$-grading and $\mathbb{Z}$-grading. The point in Definition 4.1 is that parity and weight should be considered completely independently.

EXAMPLES. 1. An arbitrary supermanifold: assign zero weight to all coordinates. 2. A vector space: assign the same (arbitrarily chosen) number $N$ as weight to all linear coordinates. 3. A vector bundle: assign $N$ for all linear fiber coordinates (even or odd) and assign zero to all base coordinates. 4. A double vector bundle, like $T E$ or $T^{*} E$ for a vector bundle $E$.

For vector spaces and vector bundles $N=1$ is a standard choice. In general, for a vector bundle $E$ we can write $E(N)$ for the choice $N$, so $E=E(1)$. Denote $E(N)^{*}:=E^{*}(-N)$. Then the natural pairing has zero weight.

TheOREM 4.1. Suppose $M$ is a graded manifold. Then the manifolds TM, $T^{*} M, T M \Pi, \Pi T^{*} M$ are bigraded. One grading is the standard vector bundle grading, the other is the induced weight.

Proof. We can lift the scaling transformations to the bundles $T M, T^{*} M$, $T M \Pi$, $\Pi T^{*} M$. If on $M$ we have $w\left(x^{a}\right)=w_{a}$, then for the natural coordinates in the fibers of $T M, T^{*} M, T M \Pi, \Pi T^{*} M$ we obtain, respectively, $w\left(v^{a}\right)=w_{a}$, $w\left(p_{a}\right)=-w_{a}, w\left(d x^{a}\right)=w_{a}, w\left(x_{a}^{*}\right)=-w_{a}$. It can be checked directly that coordinate changes respect these weights.

Various total gradings can be constructed as linear combinations of these two. 
Notice that on graded manifolds all geometric objects assume weights. In particular, for a vector field $X \in \operatorname{Vect}(M)$, if $X=X^{a}(x) \partial_{a}$, then $w(X)=w\left(X^{a}\right)-$ $w_{a}$. Thus the induced weight on $T^{*} M$ of the corresponding Hamiltonian $p(X)=$ $X^{a} p_{a}$ equals the weight of $X$ on $M$. The canonical Poisson bracket on $T^{*} M$ has induced weight zero, because the derivatives w.r.t. $x^{a}$ and $p_{a}$ have opposite weights, which mutually cancel. That means that $w(\{f, g\})=w(f)+w(g)$. It follows that the weight of the Hamiltonian vector field $X_{f}$ on $T^{*} M$ is the same as that of a function $f$. On the other hand, the fiberwise degree of the bracket on $T^{*} M$ is -1 . Similar facts hold for $\Pi T^{*} M$ and the canonical Schouten bracket.

REMARK 4.2. The topological structure of graded manifolds is an interesting question. Precisely: what kind of restrictions for a (super)manifold poses the existence of atlases with coordinate changes preserving weight.

First, it is rather obvious that if all weights are nonnegative and no "external constants" of nonzero weight (see below) are allowed, then under mild assumptions, every graded manifold can be decomposed into a tower of fibrations of the form

$$
M=M_{N} \rightarrow M_{N-1} \cdots \rightarrow M_{2} \rightarrow M_{1} \rightarrow M_{0}
$$

where coordinates on $M_{0}$ have zero weight, $M_{1} \rightarrow M_{0}$ is a vector bundle, and all other fibrations $M_{k+1} \rightarrow M_{k}$ are "affine bundles", in the sense that changes of variables are linear in fiber coordinates plus additive terms of appropriate weight. (We group all coordinates by decreasing weights $w_{N}>w_{N-1}>\ldots>w_{2}>w_{1}>$ $w_{0}=0$; at step $N$ coordinates in the fiber have weight $w_{N}$ and coordinates on the base $M_{N-1}$ have smaller weights, etc.)

Second, similarly to supermanifolds (for which we need external odd parameters or "odd constants" to be able to consider universal families, etc.), for graded manifolds we might need, in addition, to use external parameters ("constants") with assigned nonzero weights. They can enter formulas that would otherwise be not homogeneous. The role of such constants is similar to "universal constants" in physics or simply to a choice of physical units. An example of situation where this is necessary is constructing metric (of zero weight) on the total space of a vector bundle, from a metric on the base and a fiberwise metric in the bundle (which has weight -2 for the standard choice).

\section{Generalized Lie bialgebroids and their doubles}

Consider a graded $Q S$-manifold $M$. Suppose that the homological field $\hat{Q}$ has weight $q$ and the Schouten bracket $\{,\}_{S}$ has weight $s$. To them correspond odd Hamiltonians (functions on $T^{*} M$ ) $Q=p_{\hat{Q}}$ (fiberwise degree 1) and $S$ (fiberwise degree 2) of weights $q$ and $s$, respectively. To functions $Q$ and $S$ correspond Hamiltonian vector fields $X_{Q}$ and $X_{S}$ on $T^{*} M$. It is convenient to arrange the information about gradings in a table:

\begin{tabular}{|c|c|c|c|c|c|c|c|}
\hline & $\hat{Q}$ & $\{,\}_{S}$ & $Q$ & $S$ & $\{\}$, & $X_{Q}$ & $X_{S}$ \\
\hline weight on $M$ & $q$ & $s$ & - & - & - & - & - \\
\hline$w=$ induced weight on $T^{*} M$ & - & - & $q$ & $s$ & 0 & $q$ & $s$ \\
\hline$d=$ fiberwise degree & - & - & 1 & 2 & -1 & 0 & 1 \\
\hline$w+(q-s) d$ & - & - & $2 q-s$ & $2 q-s$ & $-q+s$ & $q$ & $q$ \\
\hline
\end{tabular}


The last row follows by a direct calculation.

TheOREM 5.1. Define the total weight on $T^{*} M$ as $w+(q-s) d$, where $w$ is the induced weight and $d$ the fiberwise degree. Define the vector field $\hat{Q}_{D}=X_{Q}+\lambda X_{S} \in$ $\operatorname{Vect}\left(T^{*} M\right)$ (where $\lambda \in \mathbb{R}$ is arbitrary, so it is a pencil of fields). Then $T^{*} M$ with $\hat{Q}_{D}$ is a graded $Q$-manifold; the homological vector field $\hat{Q}_{D}$ is of weight $q$, the same as the weight of $\hat{Q}$ on $M$.

Proof. Consider the Hamiltonian $Q_{D}=Q+\lambda S$. (Notice that it is odd, as it should be.) We have: $\{Q, Q\}=p([\hat{Q}, \hat{Q}])=0$ (as $\left.\hat{Q}^{2}=0\right),\{S, S\}=0$ (because $S$ defines a Schouten bracket), and $\{Q, S\}=L_{\hat{Q}} S=0$ (because $\hat{Q}$ is a derivation of the bracket). Thus $\left\{Q_{D}, Q_{D}\right\}=0$. Hence, $\left[\hat{Q}_{D}, \hat{Q}_{D}\right]=2 \hat{Q}_{D}^{2}=0$. The part concerning weights follows from the table above.

Suppose $\hat{Q}=Q^{a}(x) \partial / \partial x^{a}$ and $S=(1 / 2) S^{a b}(x) p_{b} p_{a}$. Then the explicit formula for $\hat{Q}_{D}$ is

$$
\hat{Q}_{D}=\left(Q^{a}+p_{b} S^{b a}\right) \frac{\partial}{\partial x^{a}}-(-1)^{\tilde{a}}\left(\frac{\partial Q^{b}}{\partial x^{a}} p_{b}+\frac{1}{2} \frac{\partial S^{b c}}{\partial x^{a}} p_{c} p_{b}\right) \frac{\partial}{\partial p_{a}}
$$

(we set for simplicity $\lambda=1$ ).

We denote $T^{*} M$ with the described graded $Q$-manifold structure by $D M$ and call it the double of a graded $Q S$-manifold $M$. We can consider a graded $Q S$ manifold $M$ as a generalized Lie bialgebroid (more precisely, it is a generalized antibialgebroid, i.e., a generalization of $\Pi E$ for a bialgebroid $E$ ). The case $q=$ $w(\hat{Q})=+1, s=w(S)=-1$ is the closest to the usual Lie bialgebroids.

ExAmple 5.1. Let $M=\Pi E$ for a Lie bialgebroid $E \rightarrow X$. Obviously, $D M$ as a $Q$-manifold coincides with Roytenberg's "Drinfeld's double" of the bialgebroid $E$. Let us calculate weights according to the recipe of Theorem 5.1. For $M=\Pi E$ we have the standard vector bundle weights: $w\left(x^{a}\right):=0, w\left(\xi^{i}\right):=1$ (in the notation of Section 3). The homological field has weight 1, the Schouten bracket weight -1 (see $(3.5-3.6))$. Then on $D M=T^{*} \Pi E$ we get the induced weights $w\left(p_{a}\right)=0$, $w\left(\pi_{i}\right)=-1$ and the fiberwise degree $d\left(p_{a}\right)=1, d\left(\pi_{i}\right)=1$. Hence, our general recipe for the total weight yields $w+(1-(-1)) d=w+2 d=\# \xi-\# \pi+2(\# p+\# \pi)=$ $\# \xi+\# \pi+2 \# p$, which is exactly (3.17).

The construction of the double mutatis mutandis carries over to $Q P$-manifolds. Let $M$ be a graded $Q P$-manifold with the homological field $\hat{Q}$ of weight $q$ and the Poisson bracket $\{,\}_{P}$ of weight $p$. To them correspond even multivector fields $Q^{\prime}=\theta(\hat{Q})=-Q^{a}(x) x_{a}^{*}$ (fiberwise degree 1 ) and $P=(1 / 2) P^{a b}(x) x_{b}^{*} x_{a}^{*}$ (fiberwise degree 2) of weights $q$ and $p$, respectively. Consider the supermanifold $\Pi T^{*} M$ endowed with the canonical Schouten bracket. We can again draw a table:

\begin{tabular}{|c|c|c|c|c|c|c|c|}
\hline & $\hat{Q}$ & $\{,\}_{P}$ & $Q^{\prime}$ & $P$ & $\{\}$, & $X_{Q^{\prime}}$ & $X_{P}$ \\
\hline weight on $M$ & $q$ & $p$ & - & - & - & - & - \\
\hline$w=$ induced weight on $\Pi T^{*} M$ & - & - & $q$ & $p$ & 0 & $q$ & $p$ \\
\hline$d=$ fiberwise degree & - & - & 1 & 2 & -1 & 0 & 1 \\
\hline$w+(q-p) d$ & - & - & $2 q-p$ & $2 q-p$ & $-q+p$ & $q$ & $q$ \\
\hline
\end{tabular}


Here $X_{Q^{\prime}}:=\left\{Q^{\prime},\right\}$ and $X_{P}:=\{P$,$\} stand for the odd Hamiltonian vector fields$ corresponding to the even functions $Q^{\prime}, P \in C^{\infty}\left(\Pi T^{*} M\right)$.

Theorem 5.2. Define the total weight on $\Pi T^{*} M$ as $w+(q-p) d$, where $w$ is the induced weight and $d$ the fiberwise degree. Define the vector field $\hat{Q}_{D}=$ $X_{Q^{\prime}}+\lambda X_{P} \in \operatorname{Vect}\left(\Pi T^{*} M\right)$ (where $\lambda \in \mathbb{R}$ is arbitrary). Then $\Pi T^{*} M$ with $\hat{Q}_{D}$ is a graded $Q$-manifold, and the homological vector field $\hat{Q}_{D}$ is of weight $q$, which is the same as the weight of $\hat{Q}$ on $M$.

Proof. Similar to the proof of Theorem [5.1. Consider the even function $Q_{D}^{\prime}=$ $Q^{\prime}+\lambda P \in C^{\infty}\left(\Pi T^{*} M\right)$. We have: $\left\{Q^{\prime}, Q^{\prime}\right\}=\theta([\hat{Q}, \hat{Q}])=0$ (as $\left.\hat{Q}^{2}=0\right),\{P, P\}=0$ (because $P$ defines a Poisson bracket), and $\left\{Q^{\prime}, P\right\}=L_{\hat{Q}} P=0$ (because it is equivalent to $\hat{Q}$ being a derivation of the bracket $\{,\}_{P}$, by the virtue of the non-degeneracy of the canonical Schouten bracket, cf. the proof of Theorems 2.4 and 3.3 . Thus $\left\{Q_{D}^{\prime}, Q_{D}^{\prime}\right\}=0$. Hence, $\left[\hat{Q}_{D}, \hat{Q}_{D}\right]=2 \hat{Q}_{D}^{2}=0$. The part concerning weights follows from the table.

We call the graded $Q$-manifold $\Pi T^{*} M$ the double of the graded $Q P$-manifold $M$ and denote it $D M:=\Pi T^{*} M$.

The double of a graded $Q S$-manifold is automatically a $Q P$-manifold (with respect to the canonical symplectic structure). In the same way, the double of a graded $Q P$-manifold is automatically a $Q S$-manifold, with respect to the canonical odd symplectic structure. This corresponds to the existence of a natural adinvariant inner product on Drinfeld's double $\mathfrak{d}(\mathfrak{g})=\mathfrak{g} \oplus \mathfrak{g}^{*}$. The following theorem gives partial analogs of Drinfeld's and Manin's theorems.

Theorem 5.3. Let $M$ be a graded QS-manifold with the homological field $\hat{Q}$ and the Schouten bracket $\{,\}_{S}$. The homological vector field $\hat{Q}_{D} \in \operatorname{Vect}(D M)$ on the double $D M=T^{*} M$ has the following properties:

(1) The canonical symplectic structure on $T^{*} M$ is $\hat{Q}_{D}$-invariant,

(2) $\hat{Q}_{D}$ is tangent to $M \subset D M$, and $\hat{Q}_{D \mid M}=\hat{Q}$,

(3) For arbitrary functions $f, g \in C^{\infty}(M)$ the Poisson bracket $\left\{f, \hat{Q}_{D} g\right\}$ also belongs to $C^{\infty}(M)$, and $\left\{f, \hat{Q}_{D} g\right\}=\{f, g\}_{S}$,

and it is uniquely defined by these properties. Conversely, if for a graded manifold $M$, on the cotangent bundle $T^{*} M$ there is a homological vector field $\hat{Z}$ such that it preserves the canonical Poisson bracket, is tangent to $M \subset T^{*} M$, and for arbitrary functions $f, g \in C^{\infty}(M)$ the bracket $\{f, \hat{Z} g\}$ is also a function on $M$, then $M$ is a graded $Q S$-manifold w.r.t. the vector field $\hat{Q}:=\hat{Z}_{\mid M}$ and the odd bracket defined as $\{f, g\}_{S}:=\{f, \hat{Z} g\}$.

Proof. Consider $\hat{Q}_{D}$ on the double $D M$. The statement (1) is clear. The statement (2) immediately follows from the coordinate formula (5.1). To obtain (3), notice that $\hat{Q}_{D} g=\hat{Q} g+\{S, g\}$, hence, because $\hat{Q} g \in C^{\infty}(M)$, it follows that $\left\{f, \hat{Q}_{D}\right\}=\{f,\{S, g\}\}=\{f, g\}_{S}$, for arbitrary functions $\left.f, g \in C^{\infty}(M)\right)$. Now suppose that for an arbitrary homological field $\hat{Z} \in \operatorname{Vect}(D M)$ the properties (1),(2),(3) hold. Then, by (1), we conclude that $\hat{Z}$ is Hamiltonian, with some odd Hamiltonian $Z \in C^{\infty}(D M)$, where

$$
Z=Z_{0}(x)+Z^{a}(x) p_{a}+\frac{1}{2} Z^{a b}(x) p_{b} p_{a}+\frac{1}{3 !} Z^{a b c}(x) p_{c} p_{b} p_{a} \ldots,
$$


hence

$$
\begin{aligned}
\hat{Z}=\left(Z^{a}(x)+p_{b} Z^{b a}(x)\right. & \left.+\frac{1}{2} p_{b} p_{c} Z^{c b a}(x)+\ldots\right) \frac{\partial}{\partial x^{a}} \\
& -(-1)^{\tilde{a}}\left(\frac{\partial Z_{0}}{\partial x^{a}}+\frac{\partial Z^{b}}{\partial x^{a}} p_{b}+\frac{1}{2} \frac{\partial Z^{b c}}{\partial x^{a}} p_{c} p_{b}+\ldots\right) \frac{\partial}{\partial p_{a}}
\end{aligned}
$$

From the condition (2) we conclude that $\partial Z_{0} / \partial x^{a}=0$, hence w.l.o.g. $Z_{0}$ can be set to zero, and that $Z^{a}=Q^{a}$. Taking $\{f, \hat{Z} g\}=\{f,\{Z, g\}\}$ for arbitrary functions on $M$ and applying the condition (3), we deduce that no terms of order $>2$ can be present in $(5.2)$ and that $Z^{b a}=S^{b a}$. This proves the uniqueness of $\hat{Q}_{D}$. Similar argument is applicable to an arbitrary homological field $\hat{Z} \in C^{\infty}\left(T^{*} M\right)$ with the stated properties (without assuming a given $Q S$-structure on $M$ ). We get a field $\hat{Q}:=\hat{Z}_{\mid M} \in \operatorname{Vect}(M)$ and a bracket $\{f, g\}_{S}:=\{f, \hat{Z} g\}$. That they make a $Q S$ structure follows directly.

Similar theorem holds for $Q P$-manifolds.

Now, following the example of Lie bialgebras, we want to provide the doubles of $Q S$ - and $Q P$-manifolds with brackets of the same parity as the original one. E.g., for a $Q S$-manifold $M$, we want a $\hat{Q}_{D}$-invariant Schouten bracket on $D M=T^{*} M$. To this end, recall the construction of the Schouten bracket on $T^{*} \Pi \mathfrak{g}$ (see Theorem 2.6). We shall try to mimick it.

Let $x^{a}$ be local coordinates on $M$. Let us denote the corresponding fiber coordinates in $T^{*} M$ by $y_{a}$ now (the letter $p$ is reserved for a different use). Consider the second cotangent bundle $T^{*} T^{*} M$. Let $p_{a}$ and $q^{a}$ stand for fiber coordinates in $T^{*}\left(T^{*} M\right)$ (the conjugate momenta for $x^{a}$ and $y_{a}$, respectively). The changes of coordinates have the following form (cf. equation (7.5) in Appendix):

$$
\left\{\begin{aligned}
x^{a} & =x^{a}\left(x^{\prime}\right) \\
y_{a} & =J_{a}{ }^{a^{\prime}}\left(x^{\prime}\right) y_{a^{\prime}} \\
p_{a} & =\frac{\partial x^{a^{\prime}}}{\partial x^{a}} p_{a^{\prime}}-(-1)^{\tilde{a} \tilde{b}+\tilde{c}^{\prime}} q^{c^{\prime}} J_{c^{\prime}} b \frac{\partial J_{b} a^{\prime}}{\partial x^{a}} y_{a^{\prime}} \\
(-1)^{\tilde{a}} q^{a} & =(-1)^{\tilde{a}^{\prime}} q^{a^{\prime}} J_{a^{\prime}}{ }^{a}
\end{aligned}\right.
$$

Here $J_{a}{ }^{\prime}$ stands for the Jacobi matrix. Thus, $p_{a} q^{a}=(-1)^{\tilde{a}} q^{a} p_{a}$ is not invariant. However, if we are given a linear connection on $M$, so that for a local section of $T^{*} M$ the covariant derivative is

$$
\nabla_{a} \alpha_{b}=\partial_{a} \alpha_{b}-\Gamma_{a b}^{c} \alpha_{c}
$$

then the following "long" momentum

$$
P_{a}:=p_{a}+(-1)^{\tilde{b}(\tilde{a}+1)} q^{b} \Gamma_{a b}^{c} y_{c}=p_{a}+\Gamma_{a b}{ }^{c} y_{c} q^{b}
$$

transforms as

$$
P_{a}=\frac{\partial x^{a^{\prime}}}{\partial x^{a}} P_{a^{\prime}}
$$

by a direct computation. Hence the expression $P_{a} q^{a} \in C^{\infty}\left(T^{*} T^{*} M\right)$ is invariant. Denote it by $r$ :

$$
r=P_{a} q^{a}=p_{a} q^{a}+\Gamma_{a b}{ }^{c} y_{c} q^{b} q^{a} .
$$


Following the example of Drinfeld's double for Lie bialgebras, we want to define the Schouten tensor for $D M$ as $S_{D}:=(1 / 2)\left\{Q_{D}, r\right\}$.

In the following we assume that there is a connection of zero weight at our disposal. Then $w\left(\Gamma_{a b}^{c}\right)=w_{c}-w_{a}-w_{b}$. Hence, $w\left(\Gamma_{a b}{ }^{c} y_{c} q^{b}\right)=w_{c}-w_{a}-w_{b}-w_{c}+$ $w_{b}=-w_{a}=w\left(p_{a}\right)$ ( $w$ everywhere stands for weight on $M$ and the induced weight on natural bundles), and the long momentum $P_{a}$ is homogeneous of weight $-w_{a}$. We shall also use the total weight $W:=w+(q-s) d$ on $D M$ and the corresponding induced weight on $T^{*} D M$. Notice that $W\left(x^{a}\right)=w_{a}, W\left(y_{a}\right)=-w_{a}+q-s$, $W\left(p_{a}\right)=-w_{a}, W\left(q^{a}\right)=w_{a}-q+s$.

Proposition 5.1. The function $S_{D}=(1 / 2)\left\{Q_{D}, r\right\} \in C^{\infty}\left(T^{*} D M\right)$ is quadratic in momenta $p_{a}, q^{a}$ and has weight $W\left(S_{D}\right)=s$.

Proof. Because $r$ is quadratic, $Q_{D}$ is linear and the canonical bracket has degree -1 , the function $S_{D}$ is quadratic. Now, let us calculate weight. The weight of $Q_{D}$ is the same as that of $\hat{Q}_{D}, W\left(Q_{D}\right)=q$, and the weight of $r$ is $W(r)=$ $-w_{a}+w_{a}-q+s=-q+s$. Hence, because the canonical bracket has zero weight, $W\left(S_{D}\right)=W\left(Q_{D}\right)+W(r)=q-q+s=s$.

Thus, the odd Hamiltonian $S_{D}$ defines on the double $D M$ an "almost" Schouten bracket of weight $s$ automatically compatible with $\hat{Q}_{D}$. We shall give an explicit formula for it. To this end, we may consider $x^{a}, y_{a}, P_{a}, q^{a}$ as convenient coordinates on $T^{*} D M$ (though they are not Darboux). For a function $f \in C^{\infty}(D M)$ introduce a "covariant partial derivative"

$$
\nabla_{a} f:=\frac{\partial f}{\partial x^{a}}+\Gamma_{a b}^{c} y_{c} \frac{\partial f}{\partial y_{b}} .
$$

It transforms as a component of a covector on $M$. Notice that the partial derivative $\partial f / \partial y_{a}$ transforms as a component of a vector. We also adopt convention (usual in tensor calculus) that that any operator nabla applied to a quantity with tensor indices automatically incorporates extra Christoffel symbols.

Lemma 5.1. For an arbitrary function $f \in C^{\infty}(D M)$, the Poisson bracket of $p\left(X_{f}\right)$ with $r=P_{a} q^{a}$ is given by the formula

$$
\begin{aligned}
& \left\{p\left(X_{f}\right), r\right\}= \\
& (-1)^{\tilde{f}(\tilde{a}+\tilde{b})}\left(\nabla_{a} \nabla_{b} f-(-1)^{\tilde{b} \tilde{c}} R_{a c b}^{k} y_{k} \frac{\partial f}{\partial y_{c}}\right) q^{b} q^{a}-(-1)^{(\tilde{f}+1)(\tilde{a}+\tilde{b})} \frac{\partial^{2} f}{\partial y_{a} \partial y_{b}} P_{b} P_{a} .
\end{aligned}
$$

Here $R_{a c b}^{k}$ is the Riemann tensor; the outer $\nabla_{a}$ contains an extra Christoffel symbol for the tensor index of the inner $\nabla_{b}$.

Applying this lemma to the functions $Q=Q^{a}(x) y_{a}$ and $S=(1 / 2) S^{a b}(x) y_{b} y_{a}$, we get

THEOREM 5.4. A $\hat{Q}_{D}$-invariant almost Schouten bracket on the double DM of a graded $Q S$-manifold $M$ is given by the Hamiltonian $S_{D}=(1 / 2)\left\{Q_{D}, r\right\}$ which 
has the following explicit expression

$$
\begin{aligned}
S_{D}= & \frac{1}{2}(-1)^{\tilde{a}+\tilde{b}}\left(\left(\nabla_{a} \nabla_{b} Q^{k}-(-1)^{\tilde{b} \tilde{c}+\tilde{k}(\tilde{c}+1)} R_{a c b}^{k} Q^{c}\right) y_{k}\right. \\
& \left.+\left(\frac{1}{2} \nabla_{a} \nabla_{b} S^{k l}-(-1)^{\tilde{b} \tilde{c}+(\tilde{k}+\tilde{l})(\tilde{k}+\tilde{c})} R_{a c b}^{l} S^{k c}\right) y_{l} y_{k}\right) q^{b} q^{a} \\
& -\frac{1}{2} S^{a b}(x) P_{b} P_{a} .
\end{aligned}
$$

Here $R_{a c b}^{k}$ is the Riemann tensor and nabla denotes the covariant derivative of tensor fields on the graded manifold $M$, w.r.t. the chosen linear connection.

I skip the proofs of Lemma 5.1 and Theorem 5.4. Notice that these statements are nonlinear analogs of Lemma 2.1 and formula (2.20) for Lie bialgebras. The difference with the Lie bialgebra case is due to two independent reasons. One is a possible non-flatness of $M$, hence the necessity to use a connection and the occurrence of curvature as a result. The other difference is more substantial. In a flat situation one can replace covariant derivatives by ordinary partial derivatives. In Drinfeld's case, when $Q^{k}$ is quadratic in coordinates, the first term in (5.11) would simply recover $Q^{k}$. For a general nonlinear $Q^{k}$, it would not be so. This is a hint that we should not expect, generally, for the almost Schouten bracket given by $S_{D}$ to obey Jacobi.

EXAMPLE 5.2. Consider $M=\mathbb{R}^{1 \mid 3}$ as a graded manifold, with an even coordinate $x$ and odd coordinates $\xi^{i}$ where we assign weights as $w(x)=2$ and $w\left(\xi^{i}\right)=1$. Consider

$$
\hat{Q}=\left(x \xi^{1}+\xi^{1} \xi^{2} \xi^{3}\right) \frac{\partial}{\partial x}+\xi^{1} \xi^{3} \frac{\partial}{\partial \xi^{1}}+\left(x+\xi^{1} \xi^{2}\right) \frac{\partial}{\partial \xi^{2}} .
$$

One can directly check that $\hat{Q}^{2}=0$. The homological field $(5.12)$ specifies on $\mathbb{R}^{3 \mid 1}$ a structure of an $L_{\infty}$-algebra, which is in fact homotopy equivalent to a differential Lie superalgebra. We want to consider $M$ as a nonlinear analog of a Lie algebroid. Notice that $w(\hat{Q})=+1$. Take the identically zero Schouten bracket on $M$. Hence we have a graded $Q S$-manifold which is an analog of a Lie algebra considered as a bialgebra (with the zero cobracket). The double of $M$ is $D M=T^{*} M$ with the Hamiltonian homological vector field of weight +1

$$
\begin{gathered}
\hat{Q}_{D}=\left(x \xi^{1}+\xi^{1} \xi^{2} \xi^{3}\right) \frac{\partial}{\partial x}+\xi^{1} \xi^{3} \frac{\partial}{\partial \xi^{1}}+\left(x+\xi^{1} \xi^{2}\right) \frac{\partial}{\partial \xi^{2}}- \\
\left(\xi^{1} y+\eta_{2}\right) \frac{\partial}{\partial y}+\left(\left(x+\xi^{2} \xi^{3}\right) y+\xi^{3} \eta_{1}+\xi^{2} \eta_{2}\right) \frac{\partial}{\partial \eta_{1}}- \\
\left(\xi^{1} \xi^{3} y+\xi^{1} \eta_{2}\right) \frac{\partial}{\partial \eta_{2}}+\left(\xi^{1} \xi^{2} y-\xi^{1} \eta_{1}\right) \frac{\partial}{\partial \eta_{3}}
\end{gathered}
$$

where $y$ and $\eta_{i}$ are the conjugate momenta for $x$ and $\xi^{i}$. The "total weights" are: $W(x)=2, W\left(\xi^{i}\right)=1, W(y)=0, W\left(\eta_{i}\right)=1$ (we assumed that $w(S)=-1$ ). $\hat{Q}_{D}$ is just the Lie derivative along $\hat{Q}$. (In the linear case, this corresponds to a semidirect product Lie algebra structure in $\mathfrak{g} \oplus \mathfrak{g}^{*}$ given by the coadjoint representation.) We can construct a $\hat{Q}_{D}$-invariant almost Schouten bracket on $D M$ as outlined above. Let the momenta conjugate to $x, \xi^{i}, y, \eta_{i}$ on $T^{*} D M=T^{*} T^{*} M$ be denoted as $p, \pi_{i}$, 
$q, \varkappa^{i}$, respectively. Take a flat connection on $M$ so that the covariant derivatives w.r.t. $x, \xi^{i}$ are simply the partial derivatives. Then $r=p q+\pi_{i} \varkappa^{i}$ and either directly calculating or using Theorem 5.4 we obtain $S_{D}=(1 / 2)\left\{Q_{D}, r\right\}$ as

$$
S_{D}=-y \varkappa^{1} q-\left(\xi^{3} y+\eta_{2}\right) \varkappa^{2} \varkappa^{1}+\left(\xi^{2} y-\eta_{1}\right) \varkappa^{3} \varkappa^{1}-\xi^{1} y \varkappa^{3} \varkappa^{2},
$$

which gives the following invariant almost Schouten brackets:

$$
\begin{aligned}
\left\{y, \eta_{1}\right\}_{S_{D}} & =y \\
\left\{\eta_{1}, \eta_{2}\right\}_{S_{D}} & =-\xi^{3} y-\eta_{2} \\
\left\{\eta_{1}, \eta_{3}\right\}_{S_{D}} & =-\xi^{3} y-\eta_{1} \\
\left\{\eta_{2}, \eta_{3}\right\}_{S_{D}} & =-\xi^{1} y .
\end{aligned}
$$

Notice that they are homogeneous of weight -1 . As expected, the only non-zero brackets are between the "dual" coordinates $y, \eta_{i}$ (in the Lie algebra case we would recover the Lie-Poisson bracket on $\mathfrak{g}^{*}$ ). However, they depend on variables $\xi^{i}$ as parameters, due to the nonlinearity of the original field (5.12). The Jacobi identity fails, as $\left\{S_{D}, S_{D}\right\}_{T^{*} T^{*} M}=2\left(\left(y \xi^{3}+\eta_{2}\right) \varkappa^{1} \varkappa^{2} \varkappa^{3}-y q \varkappa^{1} \varkappa^{3}\right) \neq 0$, by a direct calculation. This can be attributed either to a bad choice of a connection or to the fact that another manifestation of our graded $Q$-manifold $M$ is a strong homotopy Lie algebra - hence it would be plausible to look for "homotopy" Schouten brackets on $D M$. In fact, both considerations can be related. As mentioned above, the $L_{\infty}$-algebra defined by $\hat{Q}$ is homotopy equivalent to an ordinary Lie superalgebra; i.e., the cubic term in $\hat{Q}$ can be killed by a nonlinear transformation. There is no problem to construct a genuine bracket on the dual of a Lie superalgebra; it should give a "homotopy" bracket on a homotopy equivalent object. Or, this amounts to a different choice of a flat connection, in "linear" coordinates on $M$ corresponding to the Lie algebra structure rather than in $x, \xi^{i}$.

The toy example above illustrates the general situation (except that in general we do not have a "linear" reference object). It points to a link between the nonlinearity and homotopy . It is probable that the double for a nonlinear $Q S$ - or $Q P$ manifold should be equipped with something like a "homotopy Schouten bracket" rather than an ordinary one. This should be explored further.

\section{Odd Lie bialgebras and the odd double}

In this section we return to the linear situation and specialize our approach for odd Lie bialgebras, which were defined in Section 2. Notice that an odd bialgebra structure can be nontrivial (with a nontrivial odd cobracket) only in the super case, for $\mathfrak{g}=\mathfrak{g}_{0} \oplus \mathfrak{g}_{1}$ where $\mathfrak{g}_{1} \neq 0$.

Let $\mathfrak{g}$ be an odd Lie bialgebra. We describe its structure using the supermanifold $\Pi \mathfrak{g}$. Let the homological vector field $\hat{Q}=(1 / 2) \xi^{j} \xi^{i} Q_{i j}^{k} \partial / \partial \xi^{k}$ correspond to the Lie superalgebra structure in $\mathfrak{g}$ and let the Poisson tensor $P=(1 / 2) \xi^{k} P_{k}^{i j} \xi_{j}^{*} \xi_{i}^{*}$ correspond to the Lie superalgebra structure in $\Pi \mathfrak{g}^{*}$. Then the Lie brackets in $\mathfrak{g}$ are given by the formula

$$
\left[e_{i}, e_{j}\right]=(-1)^{\tilde{j}} Q_{i j}^{k} e_{k},
$$

and the brackets in $\Pi \mathfrak{g}^{*}$ are given by

$$
\left[\varepsilon^{i}, \varepsilon^{j}\right]=(-1)^{\tilde{i}+1} \varepsilon^{k} P_{k}^{i j} .
$$


Here $\varepsilon^{i}=\Pi e^{i}$ where the basis $e^{i}$ is right-dual to $e_{j}$. The last formula follows from the definition of the Lie-Poisson bracket (the Poisson bracket of the elements of $\Pi \mathfrak{g}^{*}$ considered as linear functions on $\Pi \mathfrak{g}$ equals their Lie bracket) and the formulas from Section 1. It also coincides with the one given by the duality argument, see Example 7.1 in the Appendix. (Notice that $\xi^{i}=\varepsilon^{i}$, as well as $x_{i}=e_{i}$.) It is not difficult to see that the homological vector field on $\mathfrak{g}^{*}=\Pi \Pi \mathfrak{g}^{*}$ defining the bracket $(6.2)$ is $\hat{Q}^{*}=(1 / 2)(-1)^{\tilde{k}+1} P_{k}^{i j} x_{j} x_{i} \partial / \partial x_{k}$.

The odd bialgebra conditions for $\mathfrak{g}$ are

$$
\{Q, Q\}=0, \quad\{P, P\}=0, \quad\{Q, P\}=0,
$$

with the canonical Schouten brackets in $\Pi T^{*} \Pi \mathfrak{g}$, where $P$ is the Poisson tensor and $Q=\theta(\hat{Q})=-(1 / 2) \xi^{j} \xi^{i} Q_{i j}^{k} \xi_{k}^{*} \in C^{\infty}\left(\Pi T^{*} \Pi \mathfrak{g}\right)$ is the even antivector field corresponding to the homological field $\hat{Q}$. (There should be no confusion with Hamiltonians used in the previous sections.)

LEMMA 6.1. For every Lie superalgebra $\mathfrak{g}$, on its antidual space $\Pi \mathfrak{g}^{*}$ there is a naturally defined odd linear map ("odd cobracket")

$$
\delta: \Pi \mathfrak{g}^{*} \rightarrow S^{2}\left(\Pi \mathfrak{g}^{*}\right) \subset \Pi \mathfrak{g}^{*} \otimes \Pi \mathfrak{g}^{*} .
$$

If the Lie bracket is given by (6.1), then the odd cobracket for it is given by the formula

$$
\delta\left(\varepsilon^{k}\right)=-\varepsilon^{j} \otimes \varepsilon^{i} Q_{i j}^{k}=-\varepsilon^{j} \varepsilon^{i} Q_{i j}^{k}
$$

Here $\varepsilon_{j} \varepsilon_{i}=(1 / 2)\left(\varepsilon_{j} \otimes \varepsilon_{i}+(-1)^{(\tilde{\imath}+1)(\tilde{\jmath}+1)} \varepsilon_{i} \otimes \varepsilon_{j}\right)$ is the symmetric product.

Proof. The map $\delta: \Pi \mathfrak{g}^{*} \rightarrow \Pi \mathfrak{g}^{*} \otimes \Pi \mathfrak{g}^{*}$ for an arbitrary Lie superalgebra $\mathfrak{g}$ is defined as follows. If we consider the Lie bracket as the linear map $\delta^{*}: \mathfrak{g} \otimes \mathfrak{g} \rightarrow \mathfrak{g}$, then the usual adjoint gives the map $\delta^{\prime}: \mathfrak{g}^{*} \rightarrow \mathfrak{g}^{*} \otimes \mathfrak{g}^{*}$, and $\delta$ is the composition of $\delta^{\prime}$ with the natural isomorphism $\mathfrak{g}^{*} \otimes \mathfrak{g}^{*} \rightarrow \Pi \mathfrak{g}^{*} \otimes \Pi \mathfrak{g}^{*}$ and the natural (odd) isomorphism $\Pi \mathfrak{g}^{*} \rightarrow \mathfrak{g}^{*}$ (left multiplication by $\Pi$ ). In terms of bases we have:

$$
\left\langle e_{i} \otimes e_{j}, \delta^{\prime}\left(e^{k}\right)\right\rangle=\left\langle\left[e_{i}, e_{j}\right], e^{k}\right\rangle=\left\langle(-1)^{\tilde{\jmath}} Q_{i j}^{l} e_{l}, \varepsilon^{k}\right\rangle=(-1)^{\tilde{\jmath}} Q_{i j}^{k},
$$

hence $\delta^{\prime}\left(e^{k}\right)=(-1)^{\tilde{\jmath}+\tilde{\imath} \tilde{\jmath}} e^{i} \otimes e^{j} Q_{i j}^{k}=(-1)^{\tilde{\imath}+1} e^{i} \otimes e^{j} Q_{j i}^{k}$. (Notice that for the basis $e^{i}$ right-dual to $e_{i},\left\langle e_{i} \otimes e_{j}, e^{k} \otimes e^{l}\right\rangle=(-1)^{\tilde{\imath} \tilde{\jmath}} \delta_{i}{ }^{k} \delta_{j}{ }^{l}$.) Now, we have $\varepsilon^{k} \mapsto \Pi \varepsilon^{k}=$ $\Pi \Pi e^{k}=e^{k}$, and we use the canonical identification $\varepsilon^{i} \otimes \varepsilon^{j}=\Pi e^{i} \otimes \Pi e^{j}$ with $(-1)^{\tilde{\imath}} e^{i} \otimes e^{j}$. Thus, for the composition, we get $\delta: \varepsilon^{k} \mapsto e^{k} \mapsto(-1)^{\tilde{\imath}+1} e^{i} \otimes e^{j} Q_{j i}^{k} \mapsto$ $-\varepsilon^{i} \otimes \varepsilon^{j} Q_{j i}^{k}$. Or:

$$
\begin{aligned}
\delta\left(\varepsilon^{k}\right)=-\varepsilon^{j} \otimes \varepsilon^{i} Q_{i j}^{k}=- & \frac{1}{2}\left(\varepsilon^{j} \otimes \varepsilon^{i} Q_{i j}^{k}+\varepsilon^{i} \otimes \varepsilon^{j} Q_{j i}^{k}\right)= \\
& -\frac{1}{2}\left(\varepsilon^{j} \otimes \varepsilon^{i}+\varepsilon^{i} \otimes \varepsilon^{j}(-1)^{(\tilde{\imath}+1)(\tilde{\jmath}+1)}\right) Q_{i j}^{k}=-\varepsilon^{j} \varepsilon^{i} Q_{i j}^{k} .
\end{aligned}
$$

In other words, $\delta=-2 \hat{Q}$ restricted to linear functions (with $\varepsilon^{k}=\xi^{k}$ ).

Proposition 6.1. If $\mathfrak{g}$ is an odd bialgebra and the Lie bracket in $\Pi \mathfrak{g}^{*}$ is given by formula (6.2), then the odd cobracket on $\mathfrak{g}$ is an odd linear map

$$
\delta: \mathfrak{g} \rightarrow S^{2}(\mathfrak{g}) \subset \mathfrak{g} \otimes \mathfrak{g}
$$

defined by the formula

$$
\delta\left(e_{k}\right)=(-1)^{\tilde{k}} P_{k}^{i j} e_{j} \otimes e_{i}=(-1)^{\tilde{k}} P_{k}^{i j} e_{j} e_{i} .
$$


Proof. Immediately follows from Lemma 6.1. For an odd bialgebra, to obtain formula (6.7) for the odd cobracket in $\mathfrak{g}$ we simply use the remark at the end of proof for Lemma 6.1 together with the above formula for the vector field $\hat{Q}^{*}$. (Notice that $e_{i}=x_{i}$.)

EXAmPle 6.1. Consider a 1|1-dimensional Lie superalgebra $\mathfrak{g}$ with a basis $e \in$ $\mathfrak{g}_{0}, \varepsilon \in \mathfrak{g}_{1}$ such that $[\varepsilon, \varepsilon]=2 e$ (the "supersymmetry algebra"). There is an odd inner product in $\mathfrak{g}$ defined by the condition $(e, \varepsilon)=-(\varepsilon, e)=1$. One can check that this product is ad $\mathfrak{g}$-invariant. Moreover, we can use this product to obtain a cobracket on $\mathfrak{g}$ (as odd-dual to the given Lie bracket). We have

$$
\begin{aligned}
& \delta(e)=0, \\
& \delta(\varepsilon)=e \otimes e .
\end{aligned}
$$

The bracket structure in $\mathfrak{g}$ corresponds to the homological field $\hat{Q}=-x^{2} \partial / \partial \xi \in$ $\operatorname{Vect}(\mathfrak{g})$, and the odd cobracket corresponds to the Poisson tensor $P=-x\left(\xi^{*}\right)^{2}$. Clearly, the Schouten bracket $\{Q, P\}$ is zero. Hence, the cobracket (6.8),(6.9) makes $\mathfrak{g}$ into an odd bialgebra.

Now let us elaborate the construction of the odd double briefly introduced in Section 2. In the sequel we use $\mathfrak{d}(\mathfrak{g}):=\mathfrak{g} \oplus \Pi \mathfrak{g}^{*}$ as the notation for the odd double. The odd double has a structure of an odd bialgebra defined as follows.

To get the Lie bracket in $\mathfrak{d}(\mathfrak{g})$, consider the sum $Q+P \in C^{\infty}\left(\Pi T^{*} \Pi \mathfrak{g}\right)$ of multivector fields $Q$ and $P$. By the equations (6.3), it satisfies $\{Q+P, Q+P\}=$ 0 . Hence the corresponding odd Hamiltonian vector field $X_{Q+P}$ is homological. Calculating by the general formulas from Section 1 , we get

$$
\begin{aligned}
& X_{Q}=-\frac{1}{2} \xi^{j} \xi^{i} Q_{j i}^{k} \frac{\partial}{\partial \xi^{k}}-(-1)^{\tilde{k}(\tilde{\jmath}+1)} \xi_{j}^{*} \xi^{i} Q_{i k}^{j} \frac{\partial}{\partial \xi_{k}^{*}}, \\
& X_{P}=(-1)^{\tilde{\jmath}} \xi_{j}^{*} \xi^{i} P_{i}^{j k} \frac{\partial}{\partial \xi^{k}}+(-1)^{\tilde{k}(\tilde{\imath}+\tilde{\jmath}+1)} \frac{1}{2} \xi_{j}^{*} \xi_{i}^{*} P_{k}^{i j} \frac{\partial}{\partial \xi_{k}^{*}} .
\end{aligned}
$$

Define the vector field $\hat{Q}_{D}$ on $\Pi T^{*} \Pi \mathfrak{g}$ as $\hat{Q}_{D}:=-X_{Q+P}$ (notice the minus sign). It is homological; explicitly:

$$
\begin{aligned}
\hat{Q}_{D}=\frac{1}{2} \xi^{j} \xi^{i} Q_{i j}^{k} \frac{\partial}{\partial \xi^{k}}+\xi_{j}^{*} \xi^{i}\left(-(-1)^{\tilde{\jmath}} P_{i}^{j k} \frac{\partial}{\partial \xi^{k}}\right. & \left.+(-1)^{\tilde{k}(\tilde{\jmath}+1)} Q_{i k}^{j} \frac{\partial}{\partial \xi_{k}^{*}}\right)- \\
& -(-1)^{\tilde{k}(\tilde{\imath}+\tilde{\jmath}+1)} \frac{1}{2} \xi_{j}^{*} \xi_{i}^{*} P_{k}^{i j} \frac{\partial}{\partial \xi_{k}^{*}} .
\end{aligned}
$$

Conversely, the condition that $\hat{Q}_{D}^{2}=0$ is equivalent to the condition $\{Q+P, Q+$ $P\}=0$, i.e., to the odd Lie bialgebra conditions (6.3). The field $\hat{Q}_{D}$ corresponds to the Lie superalgebra structure in $\mathfrak{d}(\mathfrak{g})$. Notice that $\Pi T^{*} \Pi \mathfrak{g} \cong \Pi \mathfrak{g} \oplus \mathfrak{g}^{*}=\Pi\left(\mathfrak{g} \oplus \Pi \mathfrak{g}^{*}\right)$. Here we can identify $x_{i}=\xi_{i}^{*}$. If we translate the vector field (6.12) into the language of Lie brackets, we get the following formulas

$$
\begin{aligned}
& {\left[e_{i}, e_{j}\right]=(-1)^{\tilde{\jmath}} Q_{i j}^{k} e_{k}} \\
& {\left[e_{i}, \varepsilon^{j}\right]=P_{i}^{j k} e_{k}+\varepsilon^{k} Q_{k i}^{j}} \\
& {\left[\varepsilon^{i}, \varepsilon^{j}\right]=(-1)^{\tilde{\tau}+1} \varepsilon^{k} P_{k}^{i j}}
\end{aligned}
$$

in the odd double, by a straightforward calculation using formula (2.9)). (Hence $\mathfrak{g} \subset \mathfrak{d}(\mathfrak{g})$ and $\Pi \mathfrak{g}^{*} \subset \mathfrak{d}(\mathfrak{g})$ are subalgebras.) 
To get the odd cobracket in $\mathfrak{d}(\mathfrak{g})$, we need a Poisson bracket on the supermanifold $\Pi \mathfrak{d}(\mathfrak{g})=\Pi T^{*} \Pi \mathfrak{g}$. To this end, consider the anticotangent bundle $\Pi T^{*} \Pi T^{*} \Pi \mathfrak{g}$. To avoid confusion, on $\Pi T^{*} \Pi \mathfrak{g}$ rename $\xi_{i}^{*}=: x_{i}$, so the coordinates on $\Pi T^{*} \Pi \mathfrak{g}$ will be $\xi^{i}, x_{i}$. Denote now by $\xi_{i}^{*}, x^{* i}$ the odd conjugate momenta for the coordinates $\xi^{i}, x_{i}$ respectively. Clearly, $x^{* i}$ transforms as $\xi^{i}$, hence the odd function $\rho=x^{* i} \xi_{i}^{*}=\xi_{i}^{*} x^{* i}$ on $\Pi T^{*} \Pi T^{*} \Pi \mathfrak{g}$ is well-defined. Consider the function $P_{D}$ defined as $P_{D}=(1 / 2) L_{\hat{Q}_{D}} \rho=(1 / 2)\left\{-\theta\left(\hat{Q}_{D}\right), \rho\right\}$. It follows that $P_{D}$ is a $\hat{Q}_{D}$-invariant even bivector field on $\Pi T^{*} \Pi \mathfrak{g}$. To find it explicitly we can either prove and apply an analog of Lemma 2.1 or just calculate straightforwardly.

PROPOSITION 6.2.

$$
P_{D}=\frac{1}{2} x^{* j} x^{* i} Q_{i j}^{k} x_{k}+\frac{1}{2} \xi^{k} P_{k}^{i j} \xi_{j}^{*} \xi_{i}^{*}
$$

Proof. Consider $\rho=\xi_{j}^{*} x^{* j}$ and

$$
\begin{array}{r}
-\theta\left(\hat{Q}_{D}\right)=\frac{1}{2} \xi^{j} \xi^{i} Q_{i j}^{k} \xi_{k}^{*}+\xi_{j}^{*} \xi^{i}\left(-(-1)^{\tilde{\jmath}} P_{i}^{j k} \xi_{k}^{*}+(-1)^{\tilde{k}(\tilde{\jmath}+1)} Q_{i k}^{j} x^{* k}\right)- \\
-(-1)^{\tilde{k}(\tilde{\imath}+\tilde{\jmath}+1)} \frac{1}{2} \xi_{j}^{*} \xi_{i}^{*} P_{k}^{i j} x^{* k}
\end{array}
$$

Their Schouten bracket equals

$$
\begin{gathered}
\left\{-\theta\left(\hat{Q}_{D}\right), \rho\right\}=(-1)^{\tilde{\jmath}} \frac{\partial\left(-\theta\left(\hat{Q}_{D}\right)\right)}{\partial \xi^{j}} \frac{\partial \rho}{\partial \xi_{j}^{*}}-(-1)^{\tilde{\jmath}} \frac{\partial\left(-\theta\left(\hat{Q}_{D}\right)\right)}{\partial x_{j}} \frac{\partial \rho}{\partial x^{* j}}= \\
(-1)^{\tilde{\jmath}}\left(\xi^{i} Q_{i j}^{k} \xi_{k}^{*}+(-1)^{\tilde{l}(\tilde{\jmath}+1)} x_{i}\left(-(-1)^{\tilde{\imath}} P_{j}^{i k} \xi_{k}^{*}+(-1)^{\tilde{k}(\tilde{\imath}+1)} Q_{j k}^{i} x^{* k}\right)\right) x^{* j}- \\
(-1)^{\tilde{\jmath}}\left(\xi^{i}\left(-(-1)^{\tilde{\jmath}} P_{i}^{j k} \xi_{k}^{*}+(-1)^{\tilde{k}(\tilde{\jmath}+1)} Q_{i k}^{j} x^{* k}\right)-(-1)^{\tilde{k}(\tilde{\imath}+\tilde{\jmath}+1)} x_{i} P_{k}^{i j} x^{* k}\right) \xi_{j}^{*}= \\
(-1)^{\tilde{j}} \xi^{i} Q_{i j}^{k} \xi_{k}^{*} x^{* j}-(-1)^{\tilde{\jmath}+\tilde{k}(\tilde{\jmath}+1)} Q_{i k}^{j} x^{* k} \xi_{j}^{*} \\
-(-1)^{\tilde{\jmath}+\tilde{\imath}(\tilde{\jmath}+1)+\tilde{r}} x_{i} P_{j}^{i k} \xi_{k}^{*} x^{* j}+(-1)^{\tilde{\jmath}+\tilde{k}(\tilde{\imath}+\tilde{\jmath}+1)} x_{i} P_{k}^{i j} x^{* k} \xi_{j}^{*}+ \\
(-1)^{\tilde{\jmath}+\tilde{k}(\tilde{\imath}+1)+\tilde{\imath}(\tilde{\jmath}+1)} x_{i} Q_{j k}^{i} x^{* k} x^{* j}+\xi^{i} P_{i}^{j k} \xi_{k}^{*} \xi_{j}^{*} .
\end{gathered}
$$

After exchanging the indices $j$ and $k$ in the second and the fourth terms of the last expression, changing order of factors and calculating signs, it turns out that these terms cancel with the first and the second terms respectively. In the same way, the factors in the fifth term can be rearranged so that it completely cancels signs. Finally:

$$
\left\{-\theta\left(\hat{Q}_{D}\right), \rho\right\}=x^{* k} x^{* j} Q_{j k}^{i} x_{i}+\xi^{i} P_{i}^{j k} \xi_{k}^{*} \xi_{j}^{*},
$$

which differs from the required formula only by the notation for the indices.

Corollary 6.1. $P_{D}$ is a Poisson tensor on $\Pi \mathfrak{d}(\mathfrak{g})$.

Proof. The two terms in (6.16) have the zero canonical Schouten bracket because they do not contain conjugate variables. Each term is a Poisson tensor by itself. Indeed, the second term is the Poisson tensor on $\Pi \mathfrak{g}$. The first term equals $P^{*}=(1 / 2)(-1)^{\tilde{\imath}+\tilde{\jmath}} Q_{i j}^{k} x_{k} x^{* j} x^{* i}$, the Poisson tensor that gives the LiePoisson bracket $\left\{x_{i}, x_{j}\right\}=(-1)^{\tilde{\jmath}} Q_{i j}^{k} x_{k}$ on $\mathfrak{g}^{*}$ generated by the Lie bracket in $\mathfrak{g}$. 
Corollary 6.2. The nonvanishing Poisson brackets of coordinates on $\Pi \mathfrak{d}(\mathfrak{g})$ are:

$$
\left\{\xi^{i}, \xi^{j}\right\}_{P_{D}}=(-1)^{\tilde{\imath}+1} \xi^{k} P_{k}^{i j}, \quad\left\{x_{i}, x_{j}\right\}_{P_{D}}=(-1)^{\tilde{\jmath}} Q_{i j}^{k} x_{k} .
$$

Corollary 6.3. The odd cobracket $\delta: \mathfrak{d}(\mathfrak{g}) \rightarrow S^{2} \mathfrak{d}(\mathfrak{g}) \subset \mathfrak{d}(\mathfrak{g}) \otimes \mathfrak{d}(\mathfrak{g})$ is given by the formulas:

$$
\begin{aligned}
& \delta\left(e_{k}\right)=(-1)^{\tilde{k}} P_{k}^{i j} e_{j} e_{i}, \\
& \delta\left(\varepsilon^{k}\right)=-\varepsilon^{j} \varepsilon^{i} Q_{i j}^{k} .
\end{aligned}
$$

Hence, both $\mathfrak{g} \subset \mathfrak{d}(\mathfrak{g})$ and $\Pi \mathfrak{g}^{*} \subset \mathfrak{d}(\mathfrak{g})$ are sub-bialgebras.

It is possible to formulate everything entirely in terms of linear spaces $\mathfrak{g}$ and $\Pi \mathfrak{g}^{*}$. In the corresponding analogs of Theorem 2.1 and Theorem 2.2 , there appear odd inner products.

THEOREM 6.1. Let $\mathfrak{g}$ be an odd Lie bialgebra. Then in the vector space $\mathfrak{d}(\mathfrak{g}):=$ $\mathfrak{g} \oplus \Pi \mathfrak{g}^{*}$ there is a natural structure of an odd Lie bialgebra uniquely characterized by the following properties:

(1) $\mathfrak{g}$ and $\Pi \mathfrak{g}^{*}$ are Lie subalgebras in $\mathfrak{d}(\mathfrak{g})$,

(2) The natural odd inner product in $\mathfrak{d}(\mathfrak{g})=\mathfrak{g} \oplus \Pi \mathfrak{g}^{*}$ is ad-invariant,

(3) The odd cobracket $\delta: \mathfrak{d}(\mathfrak{g}) \rightarrow S^{2} \mathfrak{d}(\mathfrak{g}) \subset \mathfrak{d}(\mathfrak{g}) \otimes \mathfrak{d}(\mathfrak{g})$ is given by the coboundary of the (odd) invariant element $\rho=e_{i} \varepsilon^{i} \in S^{2}\left(\mathfrak{g} \oplus \Pi \mathfrak{g}^{*}\right)$.

Proof. The explicit formulas defining the odd bialgebra structure in $\mathfrak{d}(\mathfrak{g})$ are given above: (6.13 6.15) and (6.20 6.21). We shall check uniqueness and the statement concerning the cobracket. Recall that an odd symmetric inner product has the property

$$
(u, v)=(-1)^{\tilde{u}+\tilde{v}+\tilde{u} \tilde{v}}(v, u) .
$$

In $\mathfrak{g} \oplus \Pi \mathfrak{g}^{*}$ the nonvanishing inner products of the basis vectors are $\left(e_{i}, \varepsilon^{j}\right)=\delta_{i}{ }^{j}$, $\left(\varepsilon^{j}, e_{i}\right)=-\delta_{i}{ }^{j}$. The ad-invariance condition for an odd inner product means that

$$
([u, v], w)+(-1)^{\tilde{u}(\tilde{v}+1)}(v,[u, w])=0
$$

for all $u, v, w$, or

$$
([v, u], w)=(-1)^{\tilde{u}}(v,[u, w]) .
$$

Suppose $\left[e_{i}, \varepsilon^{j}\right]=f_{i}^{j k} e_{k}+\varepsilon^{k} g_{k i}^{j}$ with indeterminate coefficients $f_{i}^{j k}, g_{k i}^{j}$. Then $\left(\left[e_{i}, \varepsilon^{j}\right], \varepsilon^{k}\right)=f_{i}^{j k},\left(e_{k},\left[e_{i}, \varepsilon^{j}\right]\right)=g_{k i}^{j}$. From the ad-invariance we get: $\left(\left[e_{i}, \varepsilon^{j}\right], \varepsilon^{k}\right)=$ $(-1)^{\tilde{\jmath}+1}\left(e_{i},\left[\varepsilon^{j}, \varepsilon^{k}\right]\right)=(-1)^{\tilde{\jmath}+1}\left(e_{i},(-1)^{\tilde{\jmath}+1} \varepsilon^{l} P_{l}^{j k}\right)=P_{i}^{j k}$, and also $\left(e_{k},\left[e_{i}, \varepsilon^{j}\right]\right)=$ $(-1)^{\tilde{\imath}}\left(\left[e_{k}, e_{i}\right], \varepsilon^{j}\right)=(-1)^{\tilde{\imath}}\left((-1)^{\tilde{\imath}} Q_{k i}^{l}, \varepsilon^{j}\right)=Q_{k i}^{j}$, where we used formulas (6.13) and (6.15) that follow from the condition 1 . Hence $f_{i}^{j k}=P_{i}^{j k}, g_{k i}^{j}=Q_{k i}^{j}$, and we recover formula (6.14). The uniqueness is proved. Consider now the element $\rho=e_{i} \varepsilon^{i}=(1 / 2)\left(e_{i} \otimes \varepsilon^{i}+\varepsilon^{i} \otimes e_{i}\right)$. Its coboundary in the Lie algebra cochain complex is the function $d \rho(u)=(-1)^{\tilde{u}}\left(S^{2} \operatorname{ad} u\right)(\rho)$. Hence,

$$
\begin{aligned}
d \rho\left(e_{k}\right)= & (-1)^{\tilde{k}}\left(\left[e_{k}, e_{i}\right] \varepsilon^{i}+(-1)^{\tilde{k} \tilde{\varepsilon}} \varepsilon^{i}\left[e_{k}, e_{i}\right]\right)= \\
& (-1)^{\tilde{k}}\left((-1)^{\tilde{\imath}} Q_{k i}^{l} e_{l} \varepsilon^{i}+(-1)^{\tilde{k}} \tilde{\imath} \varepsilon^{i}\left(P_{k}^{i l} e_{l}+\varepsilon^{l} Q_{l k}^{i}\right)\right)=(-1)^{\tilde{k}} P_{k}^{i l} e_{l} e_{i},
\end{aligned}
$$


where we omitted a straightforward simplification. Similarly,

$$
\begin{gathered}
d \rho\left(\varepsilon^{k}\right)=(-1)^{\tilde{k}+1}\left(\left[\varepsilon^{k}, e_{i}\right] \varepsilon^{i}+(-1)^{(\tilde{k}+1) \tilde{\imath}} \varepsilon^{i}\left[\varepsilon^{k}, e_{i}\right]\right)= \\
(-1)^{\tilde{k}+1}\left((-1)^{\tilde{k}} \varepsilon^{i}\left[e_{i}, \varepsilon^{k}\right]+(-1)^{(\tilde{k}+1) \tilde{r}} \varepsilon^{i}\left[\varepsilon^{k}, e_{i}\right]\right)= \\
(-1)^{\tilde{k}+1}\left((-1)^{\tilde{k}} \varepsilon^{i}\left(P_{i}^{k l} e_{l}+\varepsilon^{l} Q_{l i}^{k}\right)+(-1)^{(\tilde{k}+1) \tilde{r}} \varepsilon^{i}(-1)^{\tilde{k}+1} \varepsilon^{l} P_{l}^{k i}\right)=-\varepsilon^{i} \varepsilon^{l} Q_{l i}^{k} .
\end{gathered}
$$

Thus, $d \rho=\delta$.

THEOREM 6.2. Let the vector space $\mathfrak{d}=\mathfrak{a} \oplus \mathfrak{b}$ have a structure of a Lie superalgebra with an invariant odd inner product. Suppose that the subspaces $\mathfrak{a}$ and $\mathfrak{b}$ are isotropic subalgebras in $\mathfrak{d}$. Then $\mathfrak{b} \cong \Pi \mathfrak{a}^{*}, \mathfrak{a} \cong \Pi \mathfrak{b}^{*}$, and $\mathfrak{a}, \mathfrak{b}$ are odd Lie bialgebras which are in odd duality. The Lie superalgebra structure on $\mathfrak{d}$ is isomorphic to that of the odd double either of $\mathfrak{a}$ or of $\mathfrak{b}$.

Proof. It is possible to pick bases $e_{i} \in \mathfrak{a}$ and $\varepsilon^{i} \in \mathfrak{b}, \tilde{e}_{i}=\tilde{\imath}, \tilde{\varepsilon}^{i}=\tilde{e}_{i}+1=\tilde{\imath}+1$ such that $\left(e_{i}, \varepsilon^{j}\right)=\delta_{i}{ }^{j},\left(\varepsilon^{j}, e_{i}\right)=-\delta_{i}{ }^{j}$. By an argument as in the proof of the previous theorem, we immediately recover the commutators of the basis elements in the form $(6.136 .15)$. Because we are given that $\mathfrak{d}$ is a Lie superalgebra, the vector field $\hat{Q}_{D}$ defining the brackets $(6.13-6.15)$ is homological. Hence, its coefficients obey the relations that can be written as $\{Q, Q\}=0,\{P, P\}=0,\{Q, P\}=0$, and we arrive at an odd bialgebra structure in $\mathfrak{a}$. The rest is obvious. Notice that $\mathfrak{a}$ and $\mathfrak{b}$ can be interchanged.

In the classical case of Drinfeld's Lie bialgebras, one of the immediate applications of Drinfeld's double and of the concept of Manin's triples is a natural bialgebra structure in the classical Lie algebras like $\mathfrak{g l}(n)$ or $\mathfrak{s l}(n)$ and, more generally, in symmetrizable Kac-Moody Lie algebras. Roughly speaking, these algebras turn out to be "almost" doubles, i.e, are obtained from doubles by the factorization by a Lie bialgebra ideal isomorphic to an "extra copy" of a Cartan subalgebra (see 3 for details). As it turns out, this does not carry over straightforwardly to obvious candidates for an odd Lie bialgebra such as the Lie superalgebra $\mathfrak{q}(n)$ (see below). However, we can succeed by slightly generalizing the notion of the double, as follows.

Consider a Lie (super)algebra $\mathfrak{a}$ and a Lie (super)algebra $\mathfrak{h}$. We suppose the following. Let $\mathfrak{h}$ act on $\mathfrak{a}$ by derivations, and let $\mathfrak{h}$ be endowed with an invariant inner product of parity $\alpha$. For $\alpha=0$ or $\alpha=1$ assume that there is a Lie bracket in $\mathfrak{a}^{*}$ or $\Pi \mathfrak{a}^{*}$, respectively. Let the contragredient action of $\mathfrak{h}$ on $\mathfrak{a}^{*}$ or $\Pi \mathfrak{a}^{*}$ be also by derivations. Denote $\mathfrak{b}:=\mathfrak{a}^{*}$ or $\mathfrak{b}:=\Pi \mathfrak{a}^{*}$ as appropriate. Then $\mathfrak{a} \oplus \mathfrak{h} \oplus \mathfrak{b}$ will be a potential "double of $\mathfrak{a}$ (or $\mathfrak{a} \oplus \mathfrak{h}$ ) over $\mathfrak{h}$ ". More precisely, this vector space has a natural inner product of parity $\alpha$. Require a Lie bracket on $\mathfrak{a} \oplus \mathfrak{h} \oplus \mathfrak{b}$ such that both $\mathfrak{a} \oplus \mathfrak{h}$ and $\mathfrak{b} \oplus \mathfrak{h}$ (with natural brackets) are subalgebras and the inner product is invariant. It is a straightforward check that such conditions specify the bracket uniquely (if it exists). This is completely similar, e.g., to proofs of Drinfeld's theorem or Theorem 6.1. The resulting formula for the "cross" bracket would be

$$
\left[e_{i}, e^{j}\right]= \pm Q_{i}^{j k} e_{k} \pm e^{k} Q_{k i}^{j} \pm Q_{i \mu}^{j} g^{\mu \lambda} e_{\lambda} ;
$$

here we use the notation $e^{j}$ for the dual basis either in $\mathfrak{a}^{*}$ or in $\Pi \mathfrak{a}^{*}$, and $\left[e_{i}, e_{j}\right]=$ $\pm Q_{i j}^{k} e_{k},\left[e^{i}, e^{j}\right]= \pm e^{k} Q_{k}^{i j},\left[e_{\mu}, e_{i}\right]= \pm Q_{i \mu}^{k} e_{k}$ (the last formula stands for the action of $\mathfrak{h}$ on $\mathfrak{a}$ ), and $g^{\mu \lambda}$ is the inverse Gram matrix for $\mathfrak{h}$. We call $\mathfrak{a}$ a Lie bialgebra over 
$\mathfrak{h}$ (of parity $\alpha$ ) if in $\mathfrak{a} \oplus \mathfrak{h} \oplus \mathfrak{b}$ is obtained a genuine Lie bracket. Consider an invariant element $e_{i} e^{i}$ of parity $\alpha$, where the product means either the wedge product or the symmetric product. Applying ad of elements of $\mathfrak{a} \oplus \mathfrak{h} \oplus \mathfrak{b}$, we arrive at a cobracket $\delta$ on $\mathfrak{a} \oplus \mathfrak{h} \oplus \mathfrak{b}$ (even or odd). One can see that $\delta$ vanishes on $\mathfrak{h}$ (because $\mathfrak{h}$ acts, up to a sign, by the adjoint operators on $e_{i}$ and $e^{i}$ ) and that

$$
\begin{aligned}
& \delta\left(e_{k}\right)= \pm Q_{k}^{i j} e_{i} e_{j} \pm Q_{k \mu}^{j} g^{\mu \lambda} e_{j} e_{\lambda}, \\
& \delta\left(e^{k}\right)= \pm Q_{i j}^{k} e^{i} e^{j} \pm Q_{j \mu}^{k} g^{\mu \lambda} e^{j} e_{\lambda} .
\end{aligned}
$$

Immediately follows that cobrackets $(6.28)$ and (6.29) separately satisfy co-Jacobi, because they are nothing but the cobrackets dual to the Lie brackets in $\mathfrak{b} \oplus \mathfrak{h}$ and $\mathfrak{a} \oplus \mathfrak{h}$, respectively. Hence, together we have a Lie bracket in the dual (or antidual, depending on $\alpha$ ) space to $\mathfrak{g}=\mathfrak{a} \oplus \mathfrak{h} \oplus \mathfrak{b}$. By the construction, it is an ad $\mathfrak{g}$-cocycle. Hence $\mathfrak{g}=\mathfrak{a} \oplus \mathfrak{h} \oplus \mathfrak{b}$ is a Lie bialgebra (even or odd, depending on $\alpha$ ), which is, by definition, the double of $\mathfrak{a} \oplus \mathfrak{h}$ over $\mathfrak{h}$. This argument can be reversed, leading to such a "relative double" structure in a Lie (super)algebra with an invariant inner product and an appropriate decomposition into $\mathfrak{a} \oplus \mathfrak{h} \oplus \mathfrak{b}$. In particular, the even case examples with Kac-Moody algebras (see 3) are covered by this construction. For us the main motivation is the following example (where we get an odd bialgebra).

Example 6.2. Consider the matrix Lie superalgebra $\mathfrak{q}(n)$. Recall that

$$
\mathfrak{q}(n)=\{x \in \mathfrak{g l}(n \mid n) \mid[x, I]=0\}, \text { where } I=\left(\begin{array}{ll}
0 & 1 \\
1 & 0
\end{array}\right) .
$$

It consists of even matrices of the appearance

$$
x=\left(\begin{array}{cc}
a & b \\
-b & a
\end{array}\right)
$$

and odd matrices of the appearance

$$
x=\left(\begin{array}{cc}
a & b \\
b & -a
\end{array}\right) .
$$

(For the numerical entries, $b=0$ in (6.31) and $a=0$ in (6.32).) There is an invariant odd inner product on $\mathfrak{q}(n)$, defined as

$$
(x, y)=(-1)^{\tilde{x}} \operatorname{otr}(x y),
$$

where the "odd trace" is an (essentially unique) trace on $\mathfrak{q}(n)$ given by the formula

$$
\operatorname{otr} x=\frac{1}{2} \operatorname{str}(I x)=\operatorname{tr} b
$$

There is an analog of the Cartan decomposition: $\mathfrak{q}(n)=\mathfrak{n}_{+} \oplus \mathfrak{h} \oplus \mathfrak{n}$ - into the "uppertriangular", "lower-triangular" and "diagonal" matrices. The Cartan subalgebra $\mathfrak{h} \subset \mathfrak{q}(n)$ is no longer commutative. It consists of $n$ copies of the 1|1-dimensional superalgebra considered in Example 6.1. (It is exactly the non-commutativity of $\mathfrak{h}$ that prevents to define a bialgebra structure in $\mathfrak{q}(n)$ similarly to $\mathfrak{g l}(n)$.) We can apply the above considerations and interpret $\mathfrak{q}(n)$ as a "relative" double of $\mathfrak{n}_{+} \oplus \mathfrak{h}$ over $\mathfrak{h}$. Thus $\mathfrak{q}(n)$ has a natural structure of an odd bialgebra. Direct calculations of commutators yield the following explicit formulas for the odd cobracket

$$
\delta: \mathfrak{q}(n) \rightarrow S^{2} \mathfrak{q}(n)
$$


For $k=l$ :

$$
\delta\left(e_{k k}\right)=0, \quad \delta\left(\varepsilon_{k k}\right)=0
$$

for $k<l$ :

$$
\begin{aligned}
& \delta\left(e_{k l}\right)=2 \sum_{k<i<l}\left(-e_{k i} \varepsilon_{i l}+\varepsilon_{k i} e_{i l}\right)+\left(\varepsilon_{k k}-\varepsilon_{l l}\right) e_{k l}-\left(e_{k k}-e_{l l}\right) \varepsilon_{k l}, \\
& \delta\left(\varepsilon_{k l}\right)=2 \sum_{k<i<l}\left(e_{k i} e_{i l}-\varepsilon_{k i} e_{i l}\right)+\left(e_{k k}+e_{l l}\right) e_{k l}-\left(\varepsilon_{k k}-\varepsilon_{l l}\right) \varepsilon_{k l}
\end{aligned}
$$

for $k>l$ :

$$
\begin{aligned}
& \delta\left(e_{k l}\right)=2 \sum_{l<i<k}\left(e_{k i} \varepsilon_{i l}-\varepsilon_{k i} e_{i l}\right)-\left(\varepsilon_{k k}-\varepsilon_{l l}\right) e_{k l}+\left(e_{k k}-e_{l l}\right) \varepsilon_{k l}, \\
& \delta\left(\varepsilon_{k l}\right)=2 \sum_{l<i<k}\left(-e_{k i} e_{i l}+\varepsilon_{k i} e_{i l}\right)-\left(e_{k k}+e_{l l}\right) e_{k l}+\left(\varepsilon_{k k}-\varepsilon_{l l}\right) \varepsilon_{k l} .
\end{aligned}
$$

Here we use the basis $e_{i j}, \varepsilon_{i j} \in \mathfrak{q}(n)$, where $e_{i j}=\operatorname{diag}\left(E_{i j}, E_{i j}\right) \in \mathfrak{q}(n)_{0}, \varepsilon_{i j}=$ $\operatorname{antidiag}\left(E_{i j}, E_{i j}\right) \in \mathfrak{q}(n)_{1}$, and $E_{i j}$ are the usual matrix units. Note that $\left(e_{i j}, \varepsilon_{j i}\right)=$ $-\left(\varepsilon_{i j}, e_{j i}\right)=1$ and all other inner products of basis vectors are zero. A basis in $\mathfrak{n}_{+}$ is $e_{i j}, \varepsilon_{i j}$ with $i<j$, a basis in $\mathfrak{n}_{-}$is $e_{i j}, \varepsilon_{i j}$ with $i>j$, and a basis in $\mathfrak{h}$ is $e_{i i}, \varepsilon_{i i}$.

(Notice that for $\mathfrak{q}(1)$ formulas $(\overline{6.36})$ give zero cobracket, so the odd bialgebra structure given in Example 6.1 is different from the above.)

\section{Appendix}

7.1. Cotangent bundles for dual vector bundles. Here we give a full proof of Theorem 3.2 . The following statement, for purely even vector bundles, appeared for the first time, to my knowledge, in the paper by Mackenzie and Xu [17. (Earlier Tulczyjew 25] considered the particular case of tangent bundles.)

THEOREM 7.1. For an arbitrary vector bundle $E \rightarrow M$ there is a natural diffeomorphism of the cotangent bundles $F: T^{*} E \cong T^{*} E^{*}$ which preserves the symplectic structure. It is a natural transformation in the categorical sense, and $F^{2}: T^{*} E \rightarrow T^{*} E^{* *} \cong T^{*} E$ is the transformation induced by the multiplication by -1 in the fibers of $E$. (The diffeomorphism $F$ is given by the explicit formula (7.6) below.)

Proof. Let us denote by $x^{a}$ local coordinates on $M$, by $y^{i}$ coordinates in the fiber of $E \rightarrow M$, by $y_{i}$ the corresponding coordinates in the fiber of the dual bundle $E^{*} \rightarrow M$. Then a change of coordinates in $E$ has the form

$$
\left\{\begin{array} { l } 
{ x ^ { a } = x ^ { a } ( x ^ { \prime } ) } \\
{ y ^ { i } = y ^ { i ^ { \prime } } T _ { i ^ { \prime } } { } ^ { i } ( x ^ { \prime } ) }
\end{array} \quad \left\{\begin{array}{l}
x^{a}=x^{a}\left(x^{\prime}\right) \\
y_{i}=T_{i}^{i^{\prime}}\left(x^{\prime}\right) y_{i^{\prime}}
\end{array}\right.\right.
$$

(Here $T_{i}^{i^{\prime}} T_{i^{\prime}}{ }^{j}=\delta_{i}{ }^{j}$.) Denote the conjugate momenta in $T^{*} E$ and $T^{*} E^{*}$ by $p_{a}, p_{i}$ and $p_{a}, p^{i}$, respectively. For $T^{*} E$ we obtain

$$
\left\{\begin{array}{l}
p_{a}=\frac{\partial x^{a^{\prime}}}{\partial x^{a}} p_{a^{\prime}}+\frac{\partial y^{i^{\prime}}}{\partial x^{a}} p_{i^{\prime}}=\frac{\partial x^{a^{\prime}}}{\partial x^{a}} p_{a^{\prime}}+(-1)^{\tilde{a} \tilde{\imath}} y^{k^{\prime}} T_{k^{\prime}}{ }^{i} \frac{\partial T_{i}^{i^{\prime}}}{\partial x^{a}} p_{i^{\prime}} \\
p_{i}=\frac{\partial y^{i^{\prime}}}{\partial y^{i}} p_{i^{\prime}}=T_{i}^{i^{\prime}}\left(x^{\prime}\right) p_{i^{\prime}}
\end{array}\right.
$$


and for $T^{*} E^{*}$ we obtain

$$
\left\{\begin{array}{l}
p_{a}=\frac{\partial x^{a^{\prime}}}{\partial x^{a}} p_{a^{\prime}}+\frac{\partial y_{k^{\prime}}}{\partial x^{a}} p^{k^{\prime}}=\frac{\partial x^{a^{\prime}}}{\partial x^{a}} p_{a^{\prime}}+\frac{\partial T_{k^{\prime}}{ }^{i}}{\partial x^{a}} T_{i}^{i^{\prime}} y_{i^{\prime}} p^{k^{\prime}} \\
p^{i}=\frac{\partial y_{i^{\prime}}}{\partial y_{i}} p^{i^{\prime}}=(-1)^{\tilde{\imath}\left(\tilde{\imath}+\tilde{\imath}^{\prime}\right)} T_{i^{\prime}}{ }^{i}\left(x^{\prime}\right) p^{i^{\prime}}
\end{array}\right.
$$

(We assume that $\tilde{x}^{a}=\tilde{a}, \tilde{y}^{i}=\tilde{\imath}$.) Notice that $(-1)^{\tilde{\imath}\left(\tilde{\imath}+\tilde{\imath}^{\prime}\right)} T_{i^{\prime}}{ }^{i} p^{i^{\prime}}=(-1)^{\tilde{\imath}+\tilde{\imath}^{\prime}} p^{i^{\prime}} T_{i^{\prime}}{ }^{i}$ and

$$
\frac{\partial T_{k^{\prime}}^{i}}{\partial x^{a}} T_{i}^{i^{\prime}}+(-1)^{\tilde{a}\left(\tilde{k}^{\prime}+\tilde{\imath}\right)} T_{k^{\prime}}{ }^{i} \frac{\partial T_{i}^{i^{\prime}}}{\partial x^{a}}=0
$$

hence

$\frac{\partial T_{k^{\prime}}{ }^{i}}{\partial x^{a}} T_{i}^{i^{\prime}} y_{i^{\prime}} p^{k^{\prime}}=-(-1)^{\tilde{a}\left(\tilde{k}^{\prime}+\tilde{\imath}\right)+\tilde{k}^{\prime}\left(\tilde{k}^{\prime}+\tilde{a}\right)} p^{k^{\prime}} T_{k^{\prime}}{ }^{i} \frac{\partial T_{i}^{i^{\prime}}}{\partial x^{a}} y_{i^{\prime}}=-(-1)^{\tilde{a} \tilde{\imath}+\tilde{k}^{\prime}} p^{k^{\prime}} T_{k^{\prime}}{ }^{i} \frac{\partial T_{i}^{i^{\prime}}}{\partial x^{a}} y_{i^{\prime}}$.

We see that it is possible to rewrite the change of coordinates on $T^{*} E^{*}$ as

$$
\left\{\begin{aligned}
x^{a} & =x^{a}\left(x^{\prime}\right) \\
y_{i} & =T_{i}^{i^{\prime}}\left(x^{\prime}\right) y_{i^{\prime}} \\
p_{a} & =\frac{\partial x^{a^{\prime}}}{\partial x^{a}} p_{a^{\prime}}-(-1)^{\tilde{a} \tilde{\imath}+\tilde{k}^{\prime}} p^{k^{\prime}} T_{k^{\prime}}{ }^{i} \frac{\partial T_{i}^{i^{\prime}}}{\partial x^{a}} y_{i^{\prime}} \\
(-1)^{\tilde{\imath}} p^{i} & =(-1)^{\tilde{\imath}^{\prime}} p^{i^{\prime}} T_{i^{\prime}}{ }^{i}
\end{aligned}\right.
$$

which is identical with that for $T^{*} E$ if we substitute $y_{i}:=p_{i}, p^{i}:=(-1)^{\tilde{\imath}+1} y^{i}$. Hence, we define the desired diffeomorphism $F: T^{*} E \rightarrow T^{*} E^{*}$ as

$$
\left(x^{a}, y^{i}, p_{a}, p_{i}\right) \mapsto\left(x^{a}, y_{i}, p_{a}, p^{i}\right)=\left(x^{a}, p_{i}, p_{a},-(-1)^{\tilde{\imath}} y^{i}\right) .
$$

Consider the symplectic form $d p_{a} d x^{a}+d p^{i} d y_{i}$ on $T^{*} E^{*}$. Substituting (7.6), we get $d p_{a} d x^{a}+(-1)^{\tilde{\imath}+1} d y^{i} d p_{i}=d p_{a} d x^{a}+d p_{i} d y^{i}$, which is the symplectic form on $T^{*} E$. Thus, the diffeomorphism (7.6) is a canonical transformation. Notice, finally, that formulas (7.6) define the transformation $F$ for all vector bundles. One only has to be careful with the distinction of left and right coordinates: our formulas make use of left coordinates in $E$ and the corresponding right coordinates in $E^{*}$. (If we want to change, say, a left coordinate $y^{i}$ into the right coordinate, we have to multiply both $y^{i}$ and the corresponding momentum by $(-1)^{\tilde{\imath}}$.) Rewritten in left coordinates only, the formula for $F$ becomes

$$
F_{E}:\left(x^{a}, y^{i}, p_{a}, p_{i}\right) \mapsto\left(x^{a}, \bar{y}_{i}, p_{a}, \bar{p}^{i}\right)=\left(x^{a},(-1)^{\tilde{\imath}} p_{i}, p_{a},-y^{i}\right)
$$

where bar is used to denote the left coordinates in $E^{*}$ and the respective momenta. (The natural pairing of $E$ and $E^{*}$ is $y^{i} y_{i}=(-1)^{\tilde{\imath}} y^{i} \bar{y}_{i}$.) Hence, for $F_{E^{*}}$ we will get

$$
F_{E^{*}}:\left(x^{a}, \bar{y}_{i}, p_{a}, \bar{p}^{i}\right) \mapsto\left(x^{a}, w^{i}, p_{a}, q_{i}\right)=\left(x^{a},(-1)^{\tilde{\imath}} \bar{p}^{i}, p_{a},-\bar{y}_{i}\right) .
$$

At the right hand side $w^{i}$ stand for (left) coordinates in $E^{* *}$ and $q_{i}$ for the corresponding momenta. Notice that the natural isomorphism between $E^{* *}$ and $E$ is, in left coordinates, $I_{E}:\left(x^{a}, w^{i}\right) \mapsto\left(x^{a}, y^{i}\right)=\left(x^{a},(-1)^{\tilde{\imath}} w^{i}\right)$. Hence

$$
I_{E} \circ F_{E^{*}}:\left(x^{a}, \bar{y}_{i}, p_{a}, \bar{p}^{i}\right) \mapsto\left(x^{a}, y^{i}, p_{a}, p_{i}\right)=\left(x^{a}, \bar{p}^{i}, p_{a},-(-1)^{\tilde{\imath}} \bar{y}_{i}\right) .
$$

Taking composition with $F_{E}$ and using formula $(7.7)$ we finally obtain:

$$
I_{E} \circ F_{E^{*}} \circ F_{E}:\left(x^{a}, y^{i}, p_{a}, p_{i}\right) \mapsto\left(x^{a}, y^{i}, p_{a}, p_{i}\right)=\left(x^{a},-y^{i}, p_{a},-p_{i}\right),
$$

which concludes the proof, if we identify $E^{* *}$ with $E$ with the help of $I_{E}$. 


\subsection{Analog for anticotangent bundles.}

THEOREM 7.2. For an arbitrary vector bundle $E \rightarrow M$ there is a natural diffeomorphism (in the categorical sense) of the anticotangent bundles

$$
F: \Pi T^{*} E \cong \Pi T^{*}\left(\Pi E^{*}\right),
$$

which preserves the canonical odd symplectic structure. It is given by formula (7.16) below. The square $F^{2}: \Pi T^{*} E \rightarrow \Pi T^{*}\left(\Pi\left(\Pi E^{*}\right)^{*}\right) \cong \Pi T^{*} E$ is the transformation induced by the multiplication by -1 in the fibers of $E$.

Proof. Let coordinates in $E$ be denoted, as above, by $x^{a}$ and $y^{i}$. Let $x_{a}^{*}$ and $y_{i}^{*}$ be the corresponding odd momenta. Then, similarly to (7.2), we have

$$
\left\{\begin{aligned}
x^{a} & =x^{a}\left(x^{\prime}\right) \\
y^{i} & =y^{i^{\prime}} T_{i^{\prime}}{ }^{i}\left(x^{\prime}\right) \\
x_{a}^{*} & =\frac{\partial x^{a^{\prime}}}{\partial x^{a}} x_{a^{\prime}}^{*}+(-1)^{\tilde{a} \tilde{\imath}} y^{k^{\prime}} T_{k^{\prime}}{ }^{i} \frac{\partial T_{i}^{i^{\prime}}}{\partial x^{a}} y_{i^{\prime}}^{*} \\
y_{i}^{*} & =T_{i}^{i^{\prime}}\left(x^{\prime}\right) x_{i^{\prime}}^{*}
\end{aligned}\right.
$$

Consider now the antidual bundle $\Pi E^{*}$. Let $\eta_{i}$ denote the fiber coordinates in $\Pi E^{*}$ that are right-contragredient to $y^{i}$, i.e., the form $y^{i} \eta_{i}$ gives the invariant canonical odd pairing of $E$ and $\Pi E^{*}$. Notice that $\tilde{\eta}_{i}=\tilde{y}^{i}+1=\tilde{\imath}+1$. Then

$$
\eta_{i}=T_{i}^{i^{\prime}}\left(x^{\prime}\right) \eta_{i^{\prime}}=(-1)^{\left(\tilde{\imath}^{\prime}+1\right) \tilde{\imath}} \eta_{i^{\prime}} T_{i}^{i^{\prime}}\left(x^{\prime}\right)
$$

and we get

$$
\left\{\begin{aligned}
x^{a} & =x^{a}\left(x^{\prime}\right) \\
\eta_{i} & =T_{i}^{i^{\prime}}\left(x^{\prime}\right) \eta_{i^{\prime}} \\
x_{a}^{*} & =\frac{\partial x^{a^{\prime}}}{\partial x^{a}} x_{a^{\prime}}^{*}+\frac{\partial T_{k^{\prime}}{ }^{i}}{\partial x^{a}} T_{i}^{i^{\prime}} \eta_{i^{\prime}} \eta^{* k^{\prime}} \\
\eta^{* i} & =(-1)^{(\tilde{\imath}+1) \tilde{\imath}^{\prime}} T_{i^{\prime}}{ }^{i}\left(x^{\prime}\right) \eta^{* i^{\prime}}
\end{aligned}\right.
$$

for the changes of coordinates in $\Pi T^{*}\left(\Pi E^{*}\right)$. Notice that $\tilde{\eta}^{* i}=\tilde{\eta}_{i}+1=\tilde{\imath}$. Using formula (7.4) and moving $\eta^{* k^{\prime}}$ to the left, we can rewrite it as

$$
\left\{\begin{aligned}
x^{a} & =x^{a}\left(x^{\prime}\right) \\
\eta_{i} & =T_{i}^{i^{\prime}}\left(x^{\prime}\right) \eta_{i^{\prime}} \\
x_{a}^{*} & =\frac{\partial x^{a^{\prime}}}{\partial x^{a}} x_{a^{\prime}}^{*}-(-1)^{\tilde{a} \tilde{\imath}} \eta^{* k^{\prime}} T_{k^{\prime}}{ }^{i} \frac{\partial T_{i}^{i^{\prime}}}{\partial x^{a}} \eta_{i^{\prime}} \\
\eta^{* i} & =\eta^{* i^{\prime}} T_{i^{\prime}}{ }^{i}\left(x^{\prime}\right)
\end{aligned}\right.
$$

Hence, we can define the desired diffeomorphism between $\Pi T^{*} E$ and $\Pi T^{*}\left(\Pi E^{*}\right)$ by the formula

$$
F:\left(x^{a}, y^{i}, x_{a}^{*}, y_{i}^{*}\right) \mapsto\left(x^{a}, \eta_{i}, x_{a}^{*}, \eta^{* i}\right)=\left(x^{a}, y_{i}^{*}, x_{a}^{*},-y^{i}\right) .
$$

Check the symplectic form: on $\Pi T^{*}\left(\Pi E^{*}\right)$ it is $\omega=(-1)^{\tilde{a}+1} d x_{a}^{*} d x^{a}+(-1)^{\tilde{\imath}} d \eta^{* i} d \eta_{i}$, and substituting $\eta_{i}:=y_{i}^{*}, \eta^{* i}:=-y^{i}$ we get $(-1)^{\tilde{a}+1} d x_{a}^{*} d x^{a}-(-1)^{\tilde{\imath}} d y^{i} d y_{i}^{*}=$ $(-1)^{\tilde{a}+1} d x_{a}^{*} d x^{a}+(-1)^{\tilde{\imath}+1} d y_{i}^{*} d y^{i}$, which is exactly the odd symplectic form on $\Pi T^{*} E$. We omit the direct check for $F^{2}$, which is completely similar to that in the proof of Theorem 7.1 . 
EXAmple 7.1. Let $\mathfrak{g}$ be a vector space. Consider an even linear bivector field $P=(1 / 2) \xi^{k} P_{k}^{i j} \xi_{j}^{*} \xi_{i}^{*}$ on $\Pi \mathfrak{g}$. According to our theorem, there is a diffeomorphism $\Pi T^{*} \Pi \mathfrak{g} \rightarrow \Pi T^{*} \mathfrak{g}^{*}$ (preserving the odd symplectic structure), which in coordinates is given by $x_{i}=\xi_{i}^{*}, x^{* i}=-\xi^{i}$. Hence, we can transform $P$ into the function $-(1 / 2) x^{* k} P_{k}^{i j} x_{j} x_{i}=-(1 / 2)(-1)^{\tilde{k}+1} P_{k}^{i j} x_{j} x_{i} x^{* k}$ on $\Pi T^{*} \mathfrak{g}^{*}$, which is an even antivector field on $\mathfrak{g}^{*}$. Recalling $\theta$ : $\operatorname{Vect}\left(\mathfrak{g}^{*}\right) \rightarrow C^{\infty}\left(\Pi T^{*} \mathfrak{g}^{*}\right), X=X_{k}(x) \partial / \partial x_{k} \mapsto$ $(-1)^{\tilde{X}} X_{k}(x) x^{* k}$, we recover an odd vector field $\hat{Q}^{*}=(1 / 2)(-1)^{\tilde{k}+1} P_{k}^{i j} x_{j} x_{i} \partial / \partial x_{k}$. Because the symplectic structure is preserved, the field $\hat{Q}^{*}$ on $\mathfrak{g}^{*}$ is homological if and only if $P$ is a Poisson tensor.

Proposition 7.1. The Lie bracket in $\Pi \mathfrak{g}^{*}$ defined by the field $\hat{Q}^{*}$ is given by the formulas

$$
\left[\varepsilon^{i}, \varepsilon^{j}\right]=(-1)^{\tilde{\imath}+1} \varepsilon^{k} P_{k}^{i j} .
$$

Hence, the corresponding Lie-Poisson bracket on $\Pi \mathfrak{g}$ coincides with the Poisson bracket directly given by the tensor $P$.

Acknowledgements. The earlier version of this paper grew out of comments on the talks by D. Roytenberg and P. Ševera at the conference Poisson 2000 in June 2000 (CIRM, Luminy) written for myself. I thank Y. Kosmann-Schwarzbach for the invitation and for the wonderful atmosphere at the conference. This earlier version was presented at the workshop on quantization at Warwick in July 2000. I thank John Rawnsley for the invitation. I would like to mention that I first got acquainted with the problem of "Drinfeld's double" for Lie bialgebroids in 1997 at Berkeley from D. Roytenberg, who then was a Ph.D. student of Alan Weinstein. I witnessed how his work 22 came into being. It is a pleasure to mention our discussions. Also, I would like to thank H. Khudaverdian, K. Mackenzie, P. Ševera and A. Weinstein for inspiring discussions of many topics. Jim Stasheff, Kirill Mackenzie and Dmitry Roytenberg read versions of this manuscript at various stages and made many important remarks. I am deeply grateful to them.

\section{References}

[1] I. A. Batalin and G. A. Vilkovisky. Gauge algebra and quantization. Phys. Lett., 102B:27-31, 1981.

[2] I. A. Batalin and G. A. Vilkovisky. Quantization of gauge theories with linearly dependent generators. Phys. Rev., D28:2567-2582, 1983.

[3] V. Chari and A. Pressley. A guide to quantum groups. Cambridge University Press, Cambridge, 1994.

[4] M. Gerstenhaber. The cohomology structure of an associative ring. Ann. of Math., 78:267$288,1963$.

[5] Hovhannes Khudaverdian. Laplacians in odd symplectic geometry. These Proceedings, 2002.

[6] H. Khudaverdian and Th. Voronov. On odd Laplace operators. Lett. Math. Phys., 2002.

[7] O. M. Khudaverdian. Geometry of superspace with even and odd brackets. J. Math. Phys., 32:1934-1937, 1991. Preprint of the Geneva University, UGVA-DPT 1989/05-613.

[8] M. Kontsevich. Deformation quantization of Poisson manifolds, I. math.QA/9709180.

[9] Y. Kosmann-Schwarzbach. Jacobian quasi-bialgebras and quasi-Poisson Lie groups. In Mathematical aspects of classical field theory, volume 132 of Contemp. Math., pages 459-489. Amer. Math. Soc., 1992.

[10] Y. Kosmann-Schwarzbach. Exact Gerstenhaber algebras and Lie bialgebroids. Acta Appl. Math., 41:153-165, 1995.

[11] Y. Kosmann-Schwarzbach. From Poisson algebras to Gerstenhaber algebras. Ann. Inst. Fourier, Grenoble, 46:1243-1274, 1996.

[12] Y. Kosmann-Schwarzbach. Loday algebra (Leibniz algebra). In Encyclopaedia of Mathematics. Supplement II, pages 318-319. Kluwer, 2000.

[13] Tom Lada and Jim Stasheff. Introduction to SH Lie algebras for physicists. Internat. J. Theoret. Phys., 32(7):1087-1103, 1993. 
[14] P. Lecomte and C. Roger. Modules et cohomologie des bigébres de Lie. Comptes rendus Acad. Sci. Paris, 310:405-410, 1990.

[15] Zhang-Ju Liu, A. Weinstein, and Ping Xu. Manin triples for Lie bialgebroids. J. Diff. Geom., 45:547-574, 1997.

[16] J.-L. Loday. Une version non commutative des algèbres de Lie: les algèbres de Leibniz. L'Enseignement Mathématique, 39:269-293, 1993.

[17] K. C. H. Mackenzie and Ping Xu. Lie bialgebroids and Poisson groupoids. Duke Math. J., $73(2): 415-452,1994$

[18] K. C. H. Mackenzie. Lie algebroids and Lie pseudoalgebras. Bull. London Math. Soc., 27:97$147,1995$.

[19] K. C. H. Mackenzie. Drinfel'd doubles and Ehresmann doubles for Lie algebroids and Lie bialgebroids. Electronic Research Announcements of the AMS, 4:74-87, 1998.

[20] K. C. H. Mackenzie. Notions of doubles for Lie bialgebroids. 2000. Preprint.

[21] K. Mackenzie. Lie groupoids and Lie algebroids in differential geometry, volume 124 of London Mathematical Society Lecture Notes Series. Cambridge University Press, Cambridge, 1987.

[22] D. Roytenberg. Courant algebroids, derived brackets and even symplectic supermanifolds. $\mathrm{PhD}$ thesis, UC Berkeley, 1999. math.DG/9910078.

[23] J. A. Schouten. Über Differentialkomitanten zweier kontravarianter Grössen. Nederl. Akad. Wetensch., Proc., 43:449-452, 1940.

[24] Jim Stasheff. The intrinsic bracket on the deformation complex of an associative algebra. $J$. Pure Appl. Algebra, 89(1-2):231-235, 1993.

[25] W. M. Tulczyjew. A symplectic formulation of particle dynamics. In Differential Geometric Methods in Mathematical Physics, Bonn 1975, volume 570 of Lecture Notes in Math., pages 457-463. Springer-Verlag, Berlin, 1977.

[26] A. Yu. Vaŭntrob. Lie algebroids and homological vector fields. Uspekhi Matem. Nauk, 52(2):428-429, 1997.

[27] Th. Th. Voronov and A. V. Zorich. Integration on vector bundles. Funk. Anal. Pril., 22(2):14$25,1987$.

[28] Th. Th. Voronov. Class of integral transforms induced by morphisms of vector bundles. Matemat. Zametki, 44(6):735-749, 1988.

[29] Th. Th. Voronov. Quantization on supermanifolds and the analytic proof of the Atiyah-Singer index theorem, volume 38 of Sovrem. Problemy Matem. Noveřshie Dostizh. VINITI, Moscow, 1991. 3-118. [English transl. in: J. Soviet Math., 64(4):993-1069, 1993.].

[30] Th. Th. Voronov. Geometry of supermanifolds and its applications, 1992-93. Lectures at the Moscow State University.

[31] Th. Th. Voronov. On natural brackets in differential geometry and structures generated by them. Unpublished manuscript, 1993-94.

[32] Th. Th. Voronov. On the Poisson envelope of a Lie algebra. "Noncommutative" moment space. Funk. Anal. Pril., 29(3):61-64, 1995.

[33] P. Ševera. Courant algebroids and variational problems, June 2000. Colloque International "Poisson 2000". CIRM, Luminy. math.SG/0105080.

[34] Alan Weinstein. The modular automorphism group of a Poisson manifold. J. Geom. Phys., 23(3-4):379-394, 1997.

[35] A. Weinstein. Lagrangian mechanics and groupoids. In Mechanics Day, volume 7 of Fields Institute Proc. AMS, 1995.

Department of Mathematics, University of Manchester Institute of Science and

Technology (UMist), PO Box 88, Manchester M60 1QD, England

E-mail address: theodore.voronov@umist.ac.uk 